\title{
Allosteric linkages that emulate a molecular motor enzyme
}

\author{
Tosan Omabegho
}

\begin{abstract}
Allosteric mechanisms are fundamental to the operation of biomolecular motors. Recreating the molecular phenomena associated with allostery, from first principles, would help advance the design and construction of synthetic molecular motors, which remain quite simple compared natural motors. In this study, I present a model for generating allosteric interactions using mechanical linkages, which are devices in which flexible nodes are connected by rigid rods. I describe how allosteric information can be communicated between multivalent binding sites on an enzyme when linkages bind or dissociate in a stepwise fashion, which takes place stochastically according to assigned binding rates and partitioned binding energies. This design allows geometric competitions to autonomously push a linkage enzyme through a desired sequence of states, driven by consumption of a fuel. I use the model to emulate the chemical and conformational cycle of a myosin monomer, which is demonstrated by simulating chemical reaction networks of the linkage structures, and by describing how two linkage monomers can be connected together to construct a motor that walks on a track. This work shows how the complex behavior of biomolecular motors can be recapitulated with simple geometric and chemical principles that encode allosteric mechanisms. Because the concepts are material-agnostic, they can potentially be used to design and construct allosteric machines using various chemistries.
\end{abstract}

\section{Contents}

1 Introduction $\quad 2$

2 Conceptual framework for modeling allostery in myosin. 3

3 Allosteric linkage model $\quad 6$

3.1 Construction of a single-allosteric-unit (DIV) . . . . . . . . . . . . . . . . . . . 7

3.2 Construction of a double-allosteric-unit (TRV) . . . . . . . . . . . . . . . . . . . 12

3.3 Comparison of species in myosin, DIV and TRV . . . . . . . . . . . . . . 14

4 Simulating the myosin cycle with linkages $\quad 14$

4.1 The single-allosteric-unit (DIV) system . . . . . . . . . . . . . . . . . 16

4.2 The double-allosteric-unit (TRV) system . . . . . . . . . . . . . . . . . . . 19

4.3 Comparison of ligand activation and efficiency in the two systems . . . . . . . . 23

4.4 A closer look at the TRV trajectory . . . . . . . . . . . . . . . . . . . . . . . . . . . . . . . . . . . .

4.5 A comparison of DIV, TRV and myosin . . . . . . . . . . . . . . . . . . 26 


\section{Introduction}

Biomolecular motors convert chemical energy into directed motion at the nanoscale. Replicating this capability in synthetic systems is a long-term goal of engineers [1,2]. To achieve this goal, the mechanisms that allow molecular motors to function must be translated into buildable concepts which researchers can ideally apply to more than one kind of chemistry $[2,3]$.

One mechanism fundamental to how molecular motors operate is allostery. Allostery, which can be defined as intramolecular communication between binding sites, allows enzymes to link two or more chemically distinct processes together [4-6]. The basic function of allostery is to enable switch-like behavior in a molecule. For example, allostery allows a ligand to bind to an enzyme and cause the dissociation of another ligand from the enzyme, or conversely, cause tighter binding to an enzyme - negative and positive allosteric coupling, respectively. The switch-like ability of allosteric molecules allows them to transmit chemical information that is highly variable from one molecule to another, through successive binding and dissociation reactions [7].

To generate mechanical work from allosteric switching, molecular motors utilize the reciprocal nature of allosteric interactions: intramolecular allosteric information can be sent in both directions. Cytoskeletal motors (myosin, kinesin and dynein) use this inherent reciprocity to cyclically couple ATP hydrolysis to binding interactions with a polymer track, which results in nucleotide turnover and directed motion along the polymer track $[8,9]$. Cytoskeletal motors essentially convert chemical cycling between fuel and track, to mechanical cycles, by virtue of the polar geometry of their tracks and coordination between enzymatic units [3, 10, 11]. Although still complex, cytoskeletal motors have relatively simple chemical and mechanical behavior, which makes them good design targets to emulate with synthetic systems.

However, models of allostery in cytoskeletal motors, or any other enzymatic system, do not yet exist in forms that can be used to construct synthetic motors [12]. To incorporate allostery into the design of synthetic machines, scientists may postulate models of allostery and see what natural-like allosteric behavior can be achieved. An example of this engineering approach is the use mechanical linkages to model allosteric behavior [4, 13-15]. Linkages are mechanisms composed of rigid rods connected at flexible nodes that transmit motion from one side of a machine to another [16]. Several recent theoretical studies have used linkage structures to propagate mechanical signals in a way that mimics intramolecular allosteric signaling [13-15]. Binding sites are modelled as groups of nodes within a linkage structure, and an allosteric effect is correspondingly taken to be a change in the distance between the groups of nodes.

Intermolecular allosteric switching has also been demonstrated experimentally with linkage structures made from DNA [17-20]. A simple but effective principal is repeated in these experiments, which is that binding at one site can inhibit binding at the other site, by causing a change in the linkage geometry that pulls the other binding site apart. Just as in the theoretical linkage work, a binding site is something that can be divided into parts which move relative to one another in response to a conformational change of the linkage, allowing specificity at the site to be modulated. 
While the switch-like allosteric activities demonstrated in these studies mimics allostery in biological molecules, they are not sufficient to drive a motor cycle, because the allosteric switching is not two-way and cyclic, or in other words reciprocal and autonomous. My goal was to explore mechanisms that would enable continuous reciprocal allosteric switching, like that demonstrated by cytoskeletal motors. It has been suggested in theoretical models, and backed up by experimental evidence, that motor enzymes and other engine-like enzymes accomplish two-way switching by making completion of their driving process (e.g., ATP processing) contingent upon the completion of steps in the driven process (e.g., track binding), and vice versa [5, 21, 22]. This mutual dependency allows the two processes to remain continually linked together. In cytoskeletal motors for example, the release of ADP is triggered by track binding, which completes the catalysis process but begins the track process.

Here, I use linkages to demonstrate how cyclic and two-way allosteric switching can be accomplished by an enzyme that uses multivalent interactions to allosterically couple a fuel process to a ligand binding process, and make completion of the two processes reciprocally dependent upon on one another. The desired chemical behavior of the linkage enzyme system is modeled after a myosin monomer. I tested the ability of the linkage system to behave like myosin by constructing a chemical reaction network of the system and running stochastic simulations. The main constraint I placed on the group of interacting linkages is that they have the same number (five) of associated molecular species as the myosin system (myosin, ATP, ADP, Pi, and actin). I analyzed simulation trajectories to verify that the system followed a similar sequence of binding and dissociation steps (bimolecular chemical reactions) as myosin. At the outset, the mechanical action of myosin is ignored. In the final section of the paper, I show how the mechanical action of myosin can also be recapitulated with the model. The goal here is not to explain allostery in real myosin, but to translate the underlying concepts into a well-defined model, and show how a system of simple geometric relationships and parts can behave like a myosin monomer - like a motor enzyme.

\section{Conceptual framework for modeling allostery in myosin.}

In cells, myosin functions as both a dimeric enzyme, as well as a more loosely coupled group of monomers. As a multimeric complex, myosin has numerous roles in producing directed motion, including muscle movement, cellular transport and division, mechano-transduction, and the maintenance of synaptic plasticity in neurons [23]. A very accessible aspect of myosin, from both an experimental and theoretical standpoint, is that it is fully functional as a monomeric enzyme. 


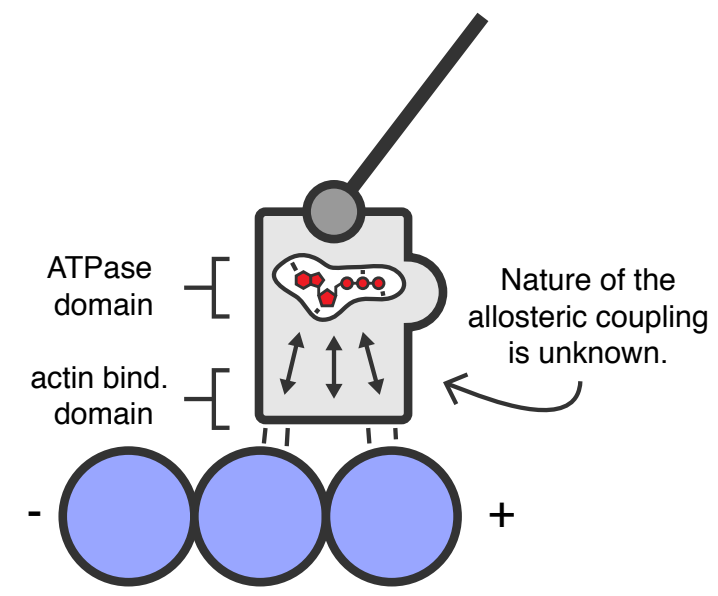

Details ignored at the outset:

1. Lever arm \& mechanical states

2. Filament structure of actin

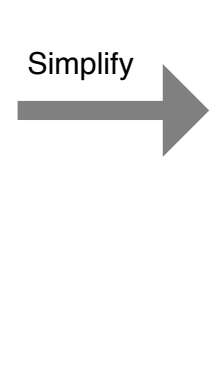

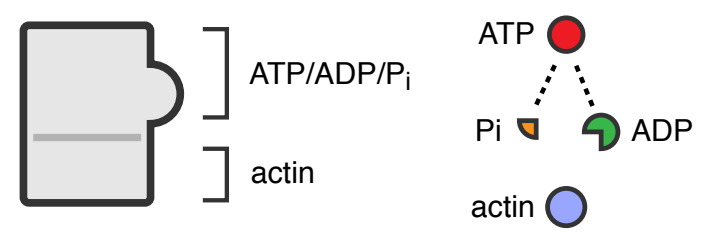

Details/concepts retained:

1. Two binding domains

2. Multivalent interactions

3. Allosteric coupling between sites

Fig. 1. Simplifying myosin. Left: A myosin monomer has a binding site for ATP (ATPase domain) and a binding site for actin (actin binding domain) that are allosterically coupled to one another. The bound state of the motor controls the angle of the lever arm domain, which points either towards the $(+)$ end, or (-) end of the actin polymer (three blue spheres). Right: The two main ways the myosin system was simplified was by ignoring the lever arm and representing actin as a ligand, rather than a linear polymer. The two allosterically coupled binding sites were still required to make multivalent associations with ATP and actin.

Myosin has two binding domains that are allosterically coupled to one another: an ATPase domain, where ATP, ADP, and Pi bind; and an actin binding domain, where actin binds (Fig. 1). Allosteric interactions between these two domains control the bound state of myosin and the angle at which the lever arm domain emerges from myosin [24]. How the structure of myosin allows allosteric information to be communicated between these domains is not well understood. Hence, a critical step I took in using linkages - which are much more simple than peptides - to model myosin, was deciding which structural details to ignore and which to retain.

I ignore two main structural details about the myosin system. The first is myosin's lever arm domain and thus the mechanical action of myosin, as mentioned above. The second is the linear geometry of the actin polymer, which forms a binding site for myosin that spans two actin subunits along the polymer. Instead, I model actin as an individual ligand that binds to a myosin monomer. The first detail I retain is that myosin has two binding sites - a binding site for ATP (and ADP and $\mathrm{Pi}$ ) and a binding site for actin. The second is that interactions at both binding sites are multivalent, meaning that each interaction is made up of multiple weaker interactions. This attribute has been verified is studies of the actin-myosin interface [25, 26] and ATP binding pocket [27-29].

The core allosteric behavior in myosin is that ATP and actin act as release factors to one another: ATP binding drives actin dissociation, whereas actin binding drives Pi and ADP dissociations. I refer to these two release factor sequences as allosteric displacements. In an allosteric displacement, a molecule binds and causes another to molecule which is bound to a different site to dissociate. It is a form of allosteric inhibition with the defined chain of events, and stands in contrast to a steric displacement, like DNA strand displacement [30], where the binding site for the displacing and displaced molecule overlap. The two allosteric displacement sequences are bridged by the hydrolysis reaction, which turns ATP into ADP and Pi. The four main reaction sequences of a 
myosin monomer are:

i The allosteric displacement of actin by ATP.

ii The cleavage of ATP into ADP and Pi (and the ligation ADP and Pi into ATP).

iii The allosteric displacement of Pi by actin.

iv The allosteric displacement of ADP by actin.

The four reaction sequences comprise the main productive cycle (productive cycle 1) of myosin, in which a single molecule of ATP drives a myosin monomer to leave one actin site and visit another. This cycle is shown on a "kinetic cube" diagram in Fig. 2 [31]. With linkages, I aimed to emulate reaction sequences i (r1 then r2 in Fig. 2), ii (r3 in Fig. 2) and iv (r4 then r6 in Fig. 2) - ignoring iii, the displacement of $\mathrm{Pi}$ by actin ( $\mathrm{r} 4$ then $\mathrm{r} 5$ in Fig. 2) - which still together define a reciprocal productive cycle (productive cycle 2) in which actin displaces product. Failures in the allosteric displacement sequences lead to futile cycles, in which ATP is not productively used. The three futile cycles discussed here, and which are represented in Fig. 2, are defined as follows:

Idling: Takes place when ATP fails to displace actin from the enzyme and cleavage takes place while the enzyme is still bound to actin, followed by the dissociation of product and the return to the actin bound state $\{$ actin $\}$.

Free-burn: This is when consecutive rounds of ATP cleavage take place without the involvement of actin. It requires that $\mathrm{Pi}$ and ADP dissociate before actin binds, leading to the empty state $(\{\varnothing\})$, followed by the binding of ATP.

Futile-reset: Takes place when actin binds to the empty state, thus like the free-burn cycle, actin has no role in displacing product.

Whereas idling is an experimentally defined cycle, the free-burn and futile-reset cycles are not - likely because of tight allosteric coupling in myosin - but, they can still be defined due to the connectivity and combinatorics of the enzymatic system. Consequently, free-burns and futile-resets have some inherent probability of taking place in a stochastic system that attempts to emulate myosin, and avoiding them in the linkage model is a central part of successful design. 
a

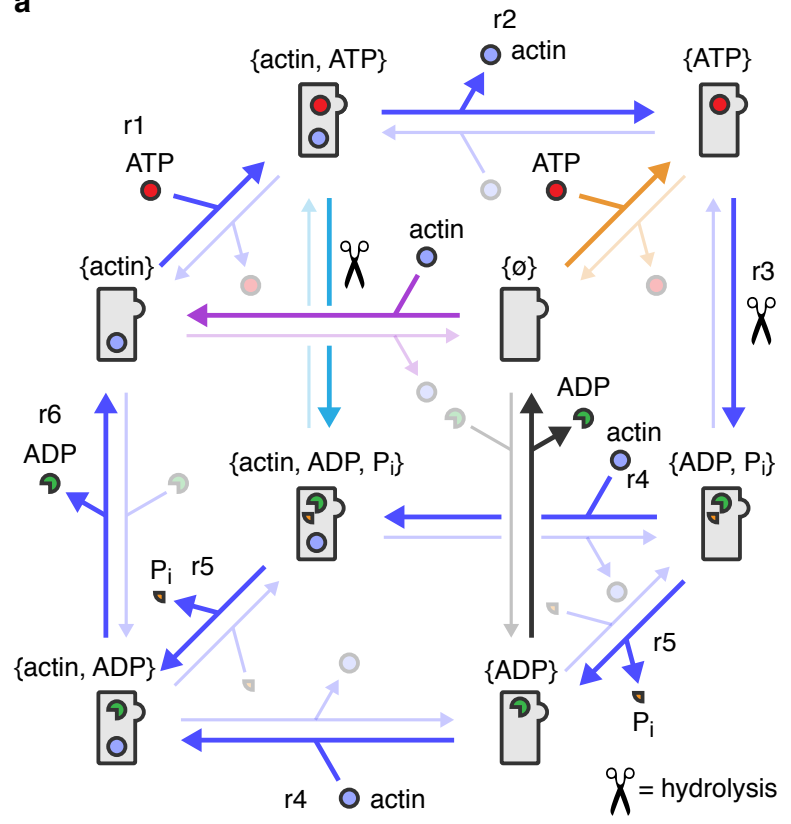

b

Goal:

Use linkages to emulate:

$\mathrm{r} 1, \mathrm{r} 2$. ATP displacing actin

r3. hydrolysis of ATP

r4,r6. actin displacing ADP
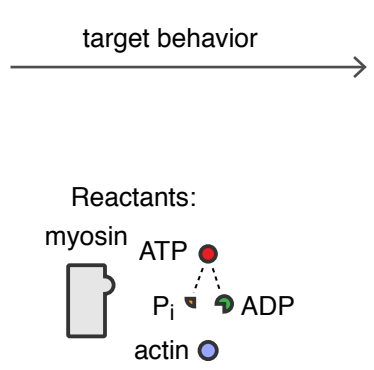

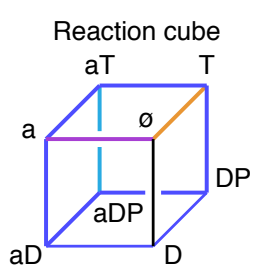

Productive cycle 2 (no displacement of $\mathrm{Pi}$ ) Productive cycle 1 (canonical)
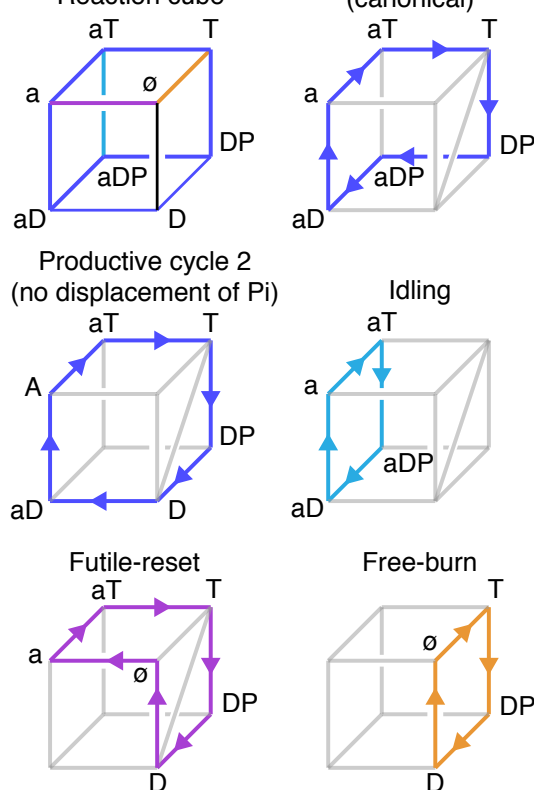

Free-burn

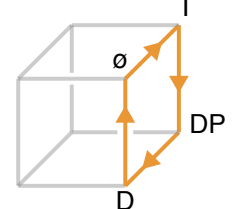

Fig. 2. Productive and futile cycles of myosin, and target behavior for linkages, displayed on a cube network. a, Network including symbolic representations of the myosin states. Bold lines denote the forward direction in each pathway (see legend in $\mathbf{b}$ ). In the canonical productive cycle (productive cycle $1)$, starting with the actin-bound state $(\{$ actin $\})$, ATP binds (r1) and allosterically displaces actin from myosin (r2), forming $\{$ ATP, actin $\}$ and then $\{$ ATP $\}$, in sequence. After the hydrolysis (r3, scissor) of ATP, which forms $\{\mathrm{ADP}, \mathrm{Pi}\}$, actin binds (r4, bottom back edge) and allosterically displaces Pi (r5, bottom left edge), and then ADP (r6), forming \{actin, ADP, Pi\} and \{actin, ADP $\}$ in sequence. The displacement of ADP returns the system to the starting actin-bound state ( $\{$ actin $\})$. In the alternative productive cycle (productive cycle 2), Pi randomly dissociates before actin binds (r5, bottom right edge), bringing the system through state $\{$ ADP $\}$. Productive cycle 2 is the target behavior for the linkage model. Three futile cycles are shown - idling (turquoise), futile-reset (purple) and free-burn (orange). Idling takes place if actin remains bound during catalysis. A futile-reset takes place if the empty-state $(\{\varnothing\})$ is reached and actin then binds. A free-burn takes place if ATP binds to the empty-state. $\mathbf{b}$, Legend showing the pathways of each productive and futile cycle separately. Abbreviations used: actin - a; ATP - T; ADP - D, Pi - P.

The general question that can be asked about the productive cycle of myosin is: what combination of mechanisms control the order in which myosin visits states in its productive cycle? Developing a linkage model for myosin was an attempt to answer this question for a synthetic system. After presenting the linkage model and the results of simulation, I review this question in section 4.5 and explicitly describe the mechanisms that drive directionality in the linkage model, and relate the answers to the myosin cycle.

\section{Allosteric linkage model}

In this section I describe how to construct systems of linkages that interact allosterically. I describe a single-allosteric-unit system and then a double-allosteric-unit system. For each system, I describe 
how to build a chemical reaction network (CRN) by enumerating all the states following certain geometric rules, and by connecting the states together using linkage reactions that are mapped to chemical reactions.

The organizing principle in the model is that binding sites are multivalent interfaces made up two to three weaker monovalent interactions, and binding is a stepwise process. Typically, the tools used to model multivalency are used to describe the interaction between a reactant comprised of multiple ligands linked together, and a receptor containing a matching set of binding sites [32, 33]. Here, I use them to model the ligand itself as the multivalent entity, and the binding site as the multivalent receptor. It is the same idea, but applied at a smaller scale.

\subsection{Construction of a single-allosteric-unit (DIV)}

To describe a single-allosteric-unit, I first define a non-enzymatic linkage domain and then an enzymatic linkage domain, and then describe how they can be combined to create a single-allostericunit system. Following this I describe how allosteric displacements can be performed with the system, and how the CRN is constructed.

To describe a non-enzymatic linkage domain, I define a mechanism whereby a multivalent interaction can be both stable and dynamic, by making two assumptions about the interaction: (1) that the individual interactions that make up the multivalent interaction are in a constant state of thermally driven dissociation and reassociation; and (2) that intramolecular binding is much faster than dissociation. Furthermore, I assume that individual interactions are chemically specific. Using these assumptions, a stable divalent interaction can be defined between a non-enzymatic linkage domain, and a ligand that binds this domain (Fig. 3). Once the ligand binds, it is probable for it to remain stably bound, mostly in the divalent state, which is rigid, but periodically transitioning to the two monovalent states, which are flexible.

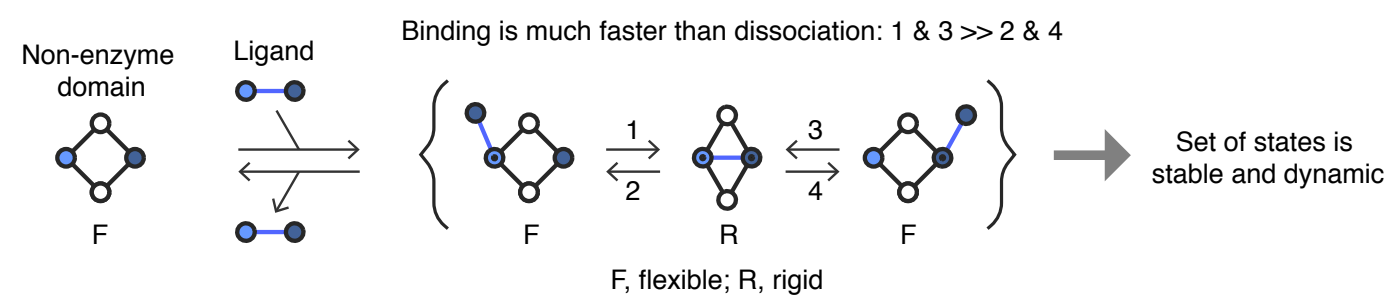

Fig. 3. Non-enzymatic linkage domain and ligand. When intramolecular binding is much faster than dissociation, the divalently binding ligand forms a stable, but dynamic set of states with the non-enzymatic domain. The complex is rigid when divalently bound, and flexible when monovalently bound.

To describe the enzymatic linkage domain, I define a linkage that can bind and cleave a fully bound substrate linkage into two products (P1 and P2); or working in reverse can bind the products and ligate them into substrate (Fig. 4). The cleavage reaction simultaneously changes the stoichiometry and rigidity of the complex by splitting the substrate linkage into two pieces, and provides a mechanism to escape the divalent stability defined for the non-enzymatic domain. Catalysis is treated as a primitive notion - there are no details to how cleavage and ligation takes place beyond the required binding states of the reactants and a rate for cleavage and ligation, which I assign to be the same in both directions. 


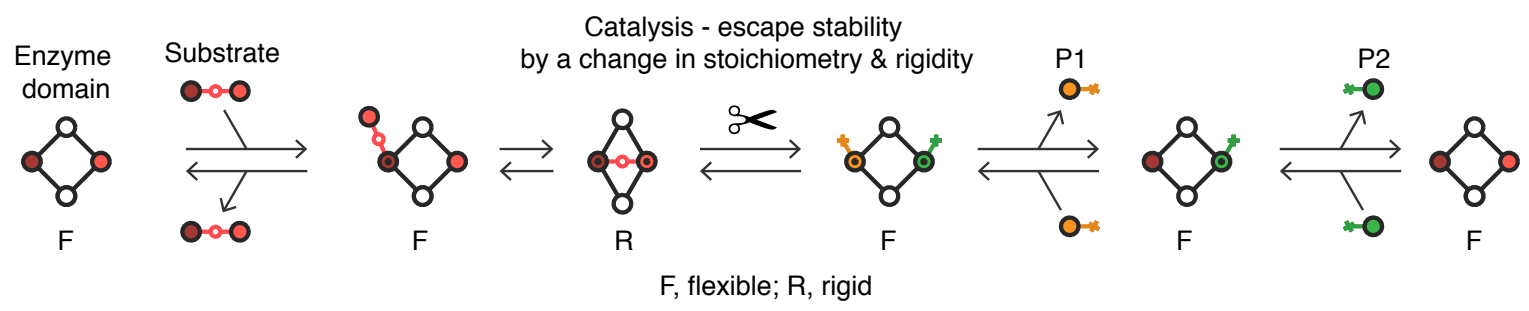

Fig. 4. Enzymatic linkage domain and substrate conversion. Upon binding divalently, the substrate can be cleaved into P1 and P2. The cleavage reaction simultaneously changes the stoichiometry and rigidity of the complex. Consequently, the stability of the complex is weakened because the monovalently bound products can dissociate randomly as a function of their individual binding energies.

To create a single-allosteric-unit, the non-enzymatic and enzymatic domains can be combined with rigid couplers to make a linkage enzyme in which the domains are geometrically coupled (Fig. $5)$. When the distance between the nodes in the one domain gets smaller, the distance between the nodes in the other domain gets larger (Fig. 5, right).
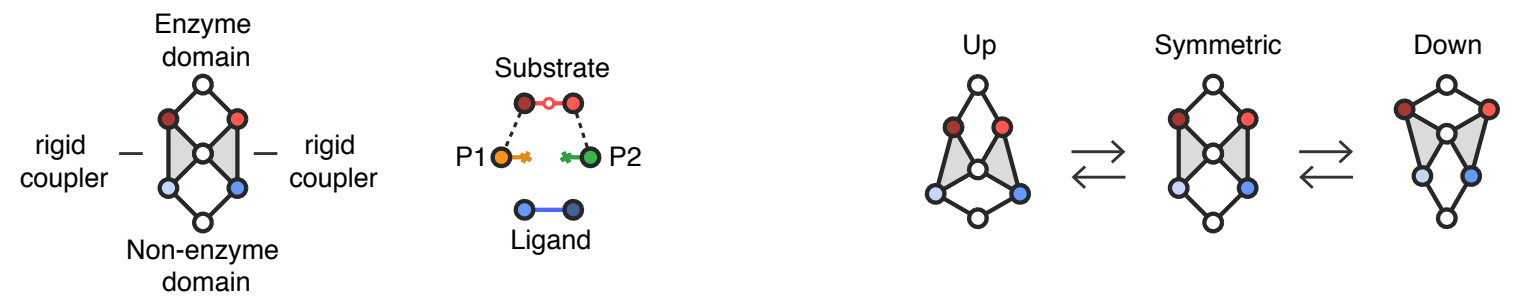

Fig. 5. An allosteric unit. Left, a non-enzymatic domain can be combined with an enzymatic linkage domain using rigid coupling domains to create an allosteric unit, named DIV. The combined allosteric unit has four reactants: S, P1, P2 and L. Right, conformational changes between the two domains are negatively coupled in the allosteric unit - when the distance between the nodes in the enzymatic domain get smaller, the distance between the nodes in the non-enzymatic domain get larger (Up), and vice versa (Down).

The bound states of the system can be enumerated using the single geometric constraint that the substrate and ligand cannot simultaneously be bound to the enzyme at two nodes. The constraint is displayed as an ' $\mathrm{x}$ ' in Fig. 6, which is an incidence matrix used to enumerate and visualize restrictions. Here I am making the first connection between a geometric constraint and an allosteric constraint. Because the ligand and substrate cannot simultaneously be bound to the enzyme at two nodes, they can be defined as being negatively allosterically coupled. By contrast, the ligand and P1, and the ligand and P2 are not coupled, because P1 and P2 both bind monovalently and cannot geometrically constrain the enzyme in any way. The states left out of Fig. 6 are states with higher stoichiometries, for example one substrate and two ligands $(\{\mathrm{S}, \mathrm{L}, \mathrm{L}\})$. The complete set of stoichiometries leads to a system with () states. 


\begin{tabular}{c|c|c|c} 
& Substrate & $\mathrm{P} 1$ & $\mathrm{P} 2$ \\
\hline \multirow{3}{*}{ Ligand } & $\begin{array}{c}\text { S, L }\} \\
\text { Negative } \\
\text { coupling }\end{array}$ & $\begin{array}{c}\text { No } 1, \mathrm{~L}\} \\
\text { coupling }\end{array}$ & $\{\mathrm{P} 1, \mathrm{~L}\}$ \\
$\mathrm{No}$ \\
coupling
\end{tabular}

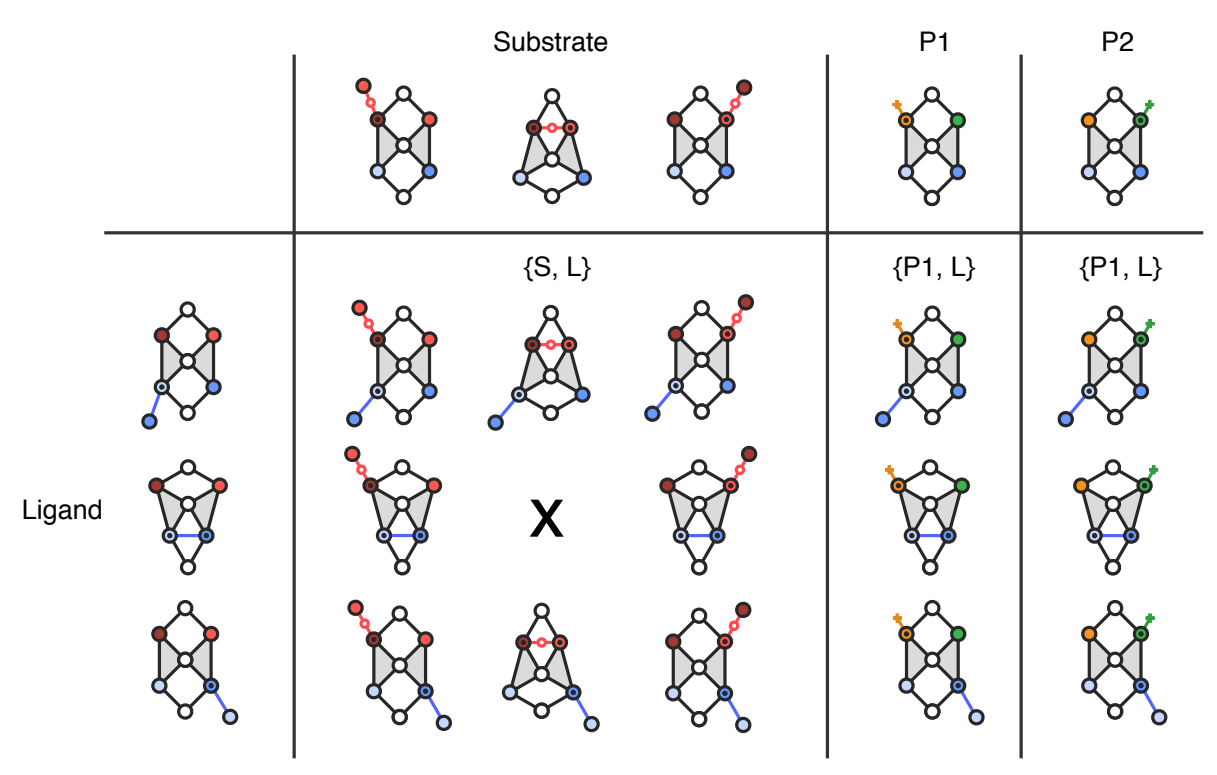

Fig. 6. Geometric restrictions used for generating the states of the system, and for emulating negative allosteric coupling. Top, coupling in words. Bottom, coupling in pictures. A single geometric restriction exits in this system, represented as an ' $\mathrm{x}$ ' in the $\{\mathrm{S}, \mathrm{L}\}$ entry, which means that the ligand and substrate cannot both be bound to the enzyme divalently.

By formally mapping changes in linkage connectivity to traditional chemical reactions, transition rates between states can be defined, and the state of the enzyme in complex with the reactants can evolve in time. The mapping:

1. Connect linkages $\rightarrow$ Bimolecular reaction

2. Separate linkages $\rightarrow$ Unimolecular reaction (dissociation)

3. Increase or decrease the connection between linkages that remain connected $\rightarrow$ Unimolecular reaction (non-dissociative)

4. Break or fuse segments (catalysis primitive) $\rightarrow$ Catalysis

To perform the mapping, each node is assigned a binding energy ( $\left.\varepsilon_{\text {node }}\right)$. The energy determines the rate of dissociation $\left(k_{d}\right)$ from a node for both a bimolecular and intramolecular transition:

$$
k_{\mathrm{d}(\text { node })}=c_{0} k_{\mathrm{bi}} e^{\beta \varepsilon_{\text {node }}}
$$

Where, $c_{0}=1 \mathrm{M}$, is a standard concentration, $k_{\mathrm{bi}}$ is a bimolecular rate constant, and $\beta=1 / k_{\mathrm{B}} T$. The intermolecular and intramolecular dissociations result in different outcomes for the composition of the enzyme complex. A dissociation from a monovalent connection (intermolecular dissociation) will reduce the number of molecules bound to the enzyme by one, whereas a dissociation from a multivalently bound molecule (intramolecular dissociation) will reduce the connectivity and rigidity 
of the complex, but not the number of molecules bound. Organizing the reactions by type leads to six different reaction types (Fig. 7). A more detailed discussion of parameter choices is given in section 4.

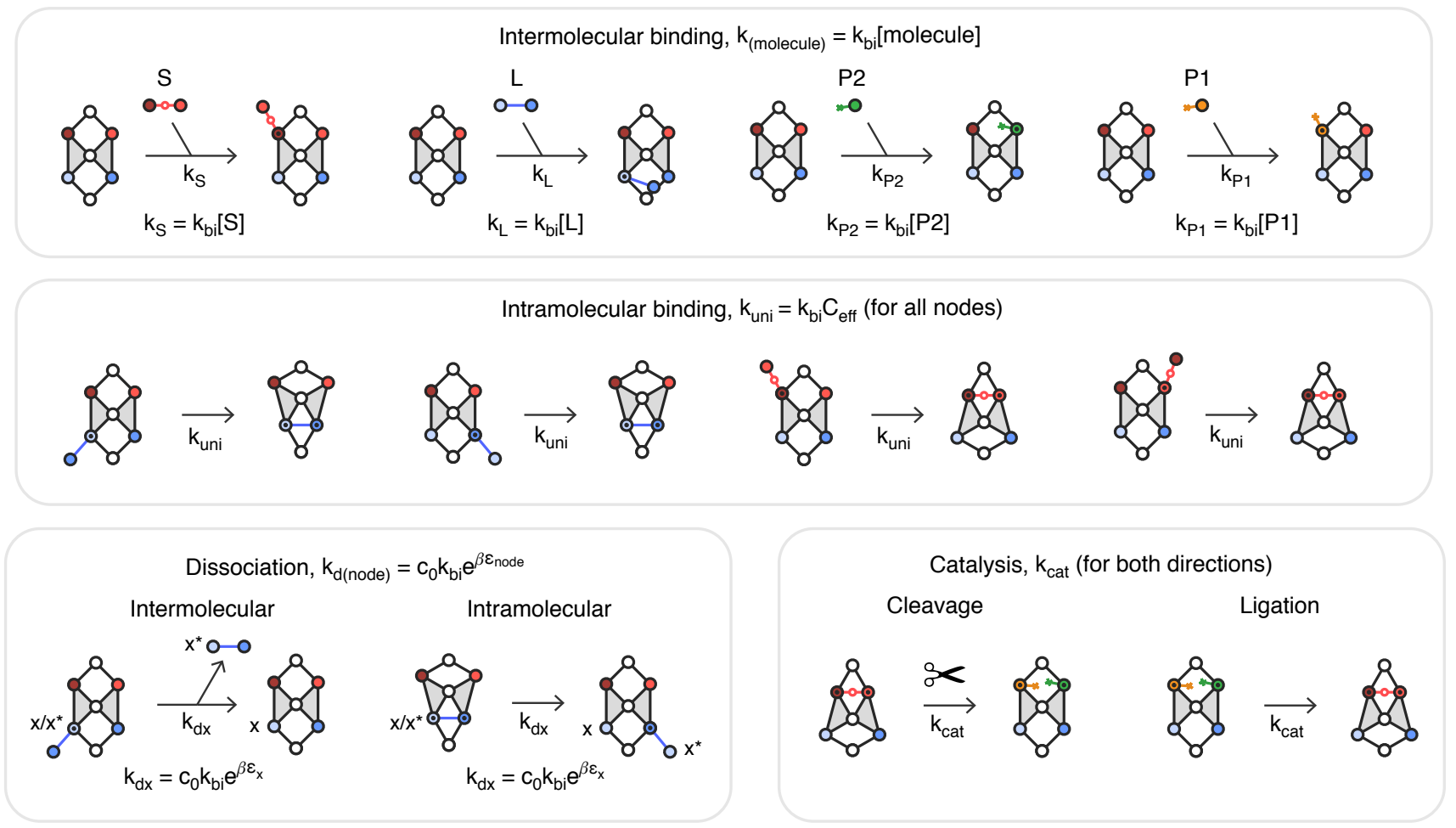

Fig. 7. The six chemical reactions - organized into four categories - that allow one linkage state to transition to another. All four categories are exhaustive in the examples shown, except Dissociation, which shows only two of the eight possible reactions that can take place: two for each of the four nodes on the enzyme. $k_{\mathrm{bi}}$ is a bimolecular rate constant. $\mathrm{C}_{\mathrm{eff}}$ is an effective intramolecular concentration. $\beta=1 / k_{\mathrm{B}} T$. $c_{0}=1 \mathrm{M}$, a standard concentration.

With the reaction types defined, I can define how the mechanism of an allosteric displacement works with linkages. In a linkage based allosteric displacement, the displacing molecule, which is geometrically coupled to molecule being displaced, wins by progressively converting the bound molecule's association with the enzyme from a stable multivalent state, to an unstable monovalent state. Displacement takes place as a random walk in which the molecule doing the displacing makes bonds only as the molecule being displaced loses bonds. And the assumption is that the displacing molecule binds tighter than the molecule being displaced. To a first approximation that ignores entropic effects, the binding energy of a molecule is the sum of its node energies:

$$
\varepsilon_{\text {molecule }} \approx \sum_{\text {node }=1}^{\mathrm{N}} \varepsilon_{\text {node }}
$$

Hence, tighter binding means:

$$
\varepsilon_{\text {displacer }}>\varepsilon_{\text {displaced }}
$$


A bi-directional displacement reaction is pictured in Fig. 8, to communicate how, all else being equal, the winner is determined by the energies assigned to the nodes.

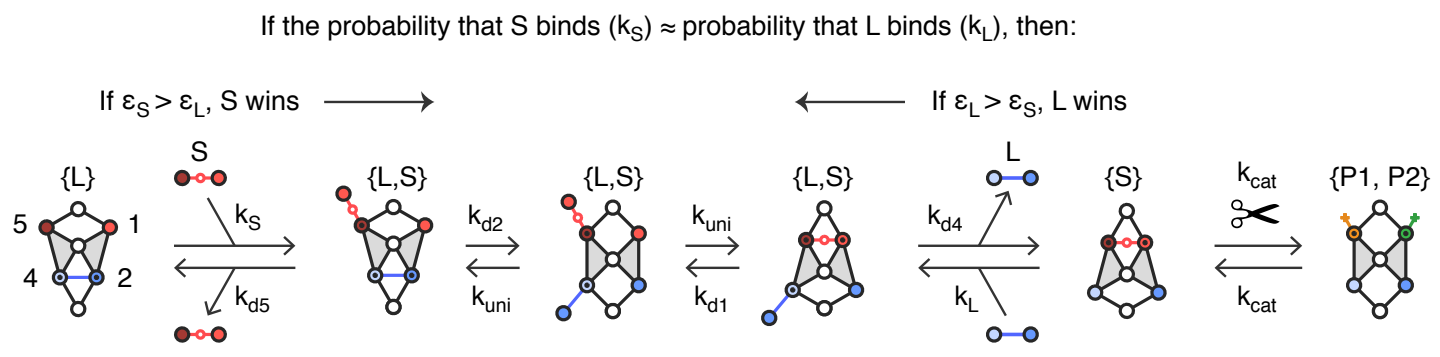

Fig. 8. Allosteric displacement with linkages. The direction of the displacement is determined by the relative binding strengths of the molecules competing with one another. If the probability of binding is roughly the same for $S$ and $L$, then if $S$ binds tighter $\left(\varepsilon_{S}>\varepsilon_{L}\right)$, it will allosterically displace $L$ from the enzyme. Conversely, if $\mathrm{L}$ binds tighter $\left(\varepsilon_{\mathrm{L}}>\varepsilon_{\mathrm{S}}\right)$, L will allosterically displace $\mathrm{S}$.

The allosteric mechanism of displacement borrows from the stepwise mechanism by which one DNA strand displaces another in a DNA strand displacement reaction [30, 34]. When two DNA strands are competing with each other for exclusive binding to a partner, a binding reaction in the "contested" region is always preceded by random dissociation from that region. Thus, a gain by one species is preceded by a random loss by the other that exposes a portion of the binding site. What I did was reimagine that sequence of loss-then-gain steps for a pair of allosterically coupled binding sites and ligands. In the allosteric version, "exposes" is redefined geometrically to mean "make reachable". Thus, upon dissociation at either of the two allosterically coupled sites, a subsequent increase in flexibility of the complex allows the competing species to physically reach a previously unreachable segment of its binding site. Like DNA strand displacement the process is mutually exclusive - a gain for one species is a loss for the other, and vice versa.

By connecting all the states that are one reaction away from one another, the complete chemical reaction network (CRN) can be defined (Fig. 9). The network consists of forty-nine states and 260 connecting reactions. Each state in the CRN is a different configuration of the enzyme in complex with the four reactants. 

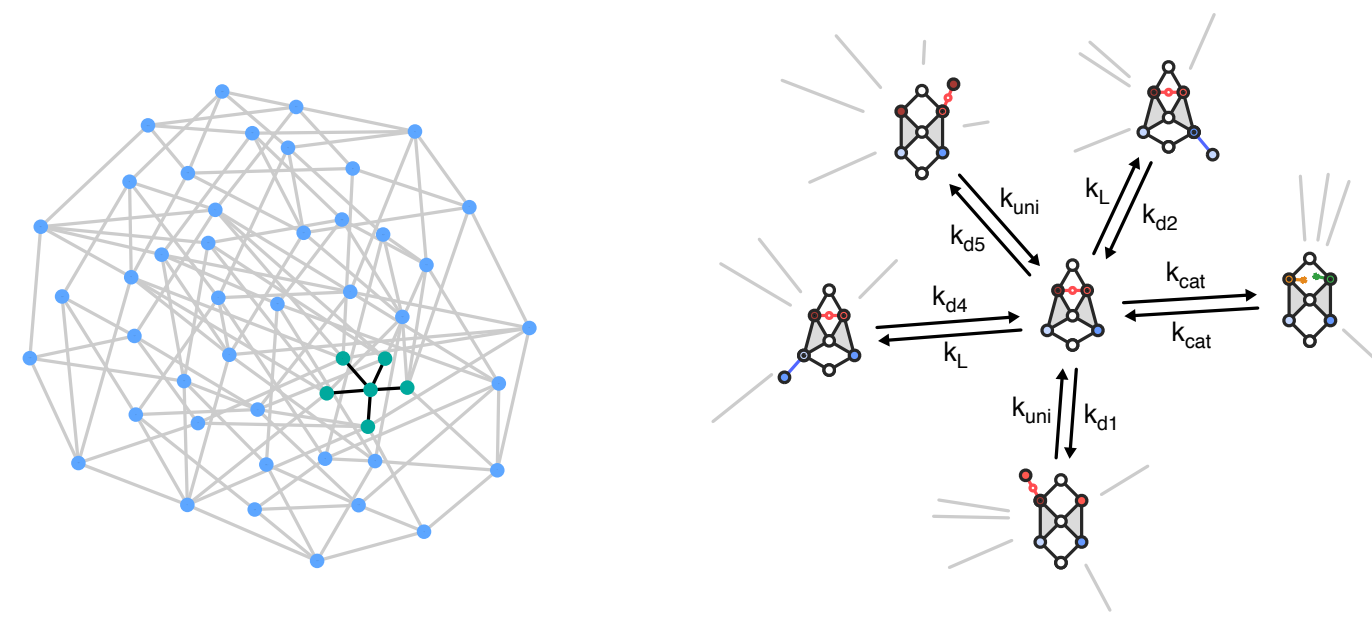

Fig. 9. Chemical reaction network of a single-allosteric-unit. Left, a graph representation of the network. The network consists of forty-nine states and 260 connections between states, where each connection is a two-way reaction. Right, the green colored nodes in the graph representation are expanded to show the states and transition rates that connect them. Each connection is represented by its forward and reverse reactions.

\subsection{Construction of a double-allosteric-unit (TRV)}

Two allosteric units can be combined to make a larger linkage enzyme, named TRV (for "TRiValent"), because the substrate and ligand binds trivalently to the enzyme (Fig. 10). An important difference in this system is that catalysis leads to asymmetric products - a smaller product that is bound monovalently and unstably (P1), and a larger product that is bound divalently and stably (P2). Cleavage only takes place in the unit on the left, but the reaction requires that the substrate is bound at all three nodes.
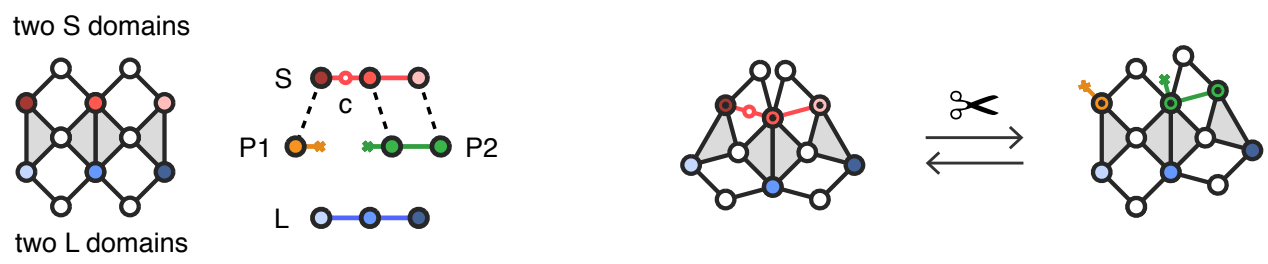

Fig. 10. A double-allosteric-unit. Left, two allosteric units can be combined to make a double-allostericunit, named TRV. Like DIV, TRV has four reactants (S, P1, P2 and L), except here P1 and P2 are different sizes. Right, the reversible cleavage/ligation reactions result in either the fully bound substrate being cleaved into P1 and P2, or P1 and P2 being ligated into substrate. After cleavage, P1 is bound monovalently, and P2 divalently.

Like for the DIV system, the bound states of the system can be enumerated using the geometric constraints of the system. Here, there two constraints: (1) the substrate and ligand cannot be simultaneously bound to the left or right allosteric units at two nodes; and (2) P2 and ligand 
cannot be simultaneously bound to the right unit at two nodes. Thus in the TRV system, the ligand is negatively allosterically coupled to both the substrate and P2.

\begin{tabular}{c|c|c|c} 
& Substrate & $\mathrm{P} 1$ & $\mathrm{P} 2$ \\
\hline \multirow{3}{*}{ Ligand } & $\begin{array}{c}\text { S }, \mathrm{L}\} \\
\text { Negative } \\
\text { coupling }\end{array}$ & $\begin{array}{c}\text { P1, L }\} \\
\text { No } \\
\text { coupling }\end{array}$ & $\begin{array}{c}\text { Negative } \\
\text { coupling }\end{array}$
\end{tabular}

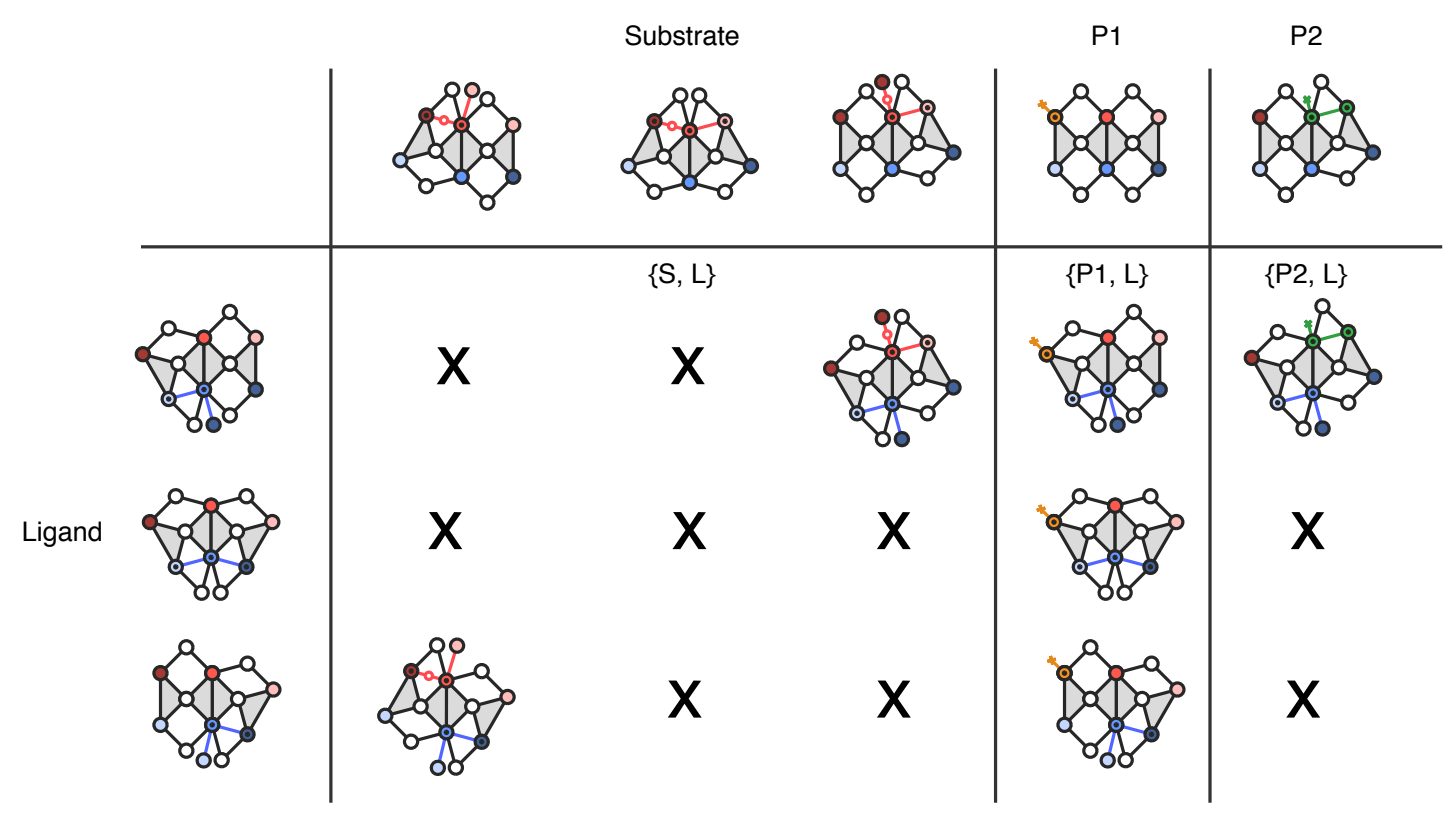

Fig. 11. Geometric restrictions used for generating the states of the system, and for emulating negative allosteric coupling. Top, coupling in words. Bottom, coupling in pictures. Geometric restrictions are multiplied for $\{\mathrm{S}, \mathrm{L}\}$ in the double-allosteric-unit because the $\mathrm{S}$ and $\mathrm{L}$ can bind trivalently, and divalenty on each side. Like DIV, $\{\mathrm{P} 1, \mathrm{~L}\}$ in TRV has no restrictions. Unlike DIV, $\{\mathrm{P} 2, \mathrm{~L}\}$ in TRV has two restrictions, because $\mathrm{P} 2$ binds divalently.

I create an ad hoc restriction named the adjacency rule to eliminate two divalent states from the total set of possible states: one in which the substrate is bound to the two outer nodes (Fig. 12, left); and one in which the ligand is bound to the two outer nodes (Fig. 12, right). Without this rule two more intramolecular rates would have to be assigned: one rate for the transition from the two outside bonds being connected to the trivalently bound state; and a second rate for one outside connected bond, to two outside connected bonds. Thus, in this study I chose to simplify the model by eliminating these states and transitions, but may include them in future studies. 

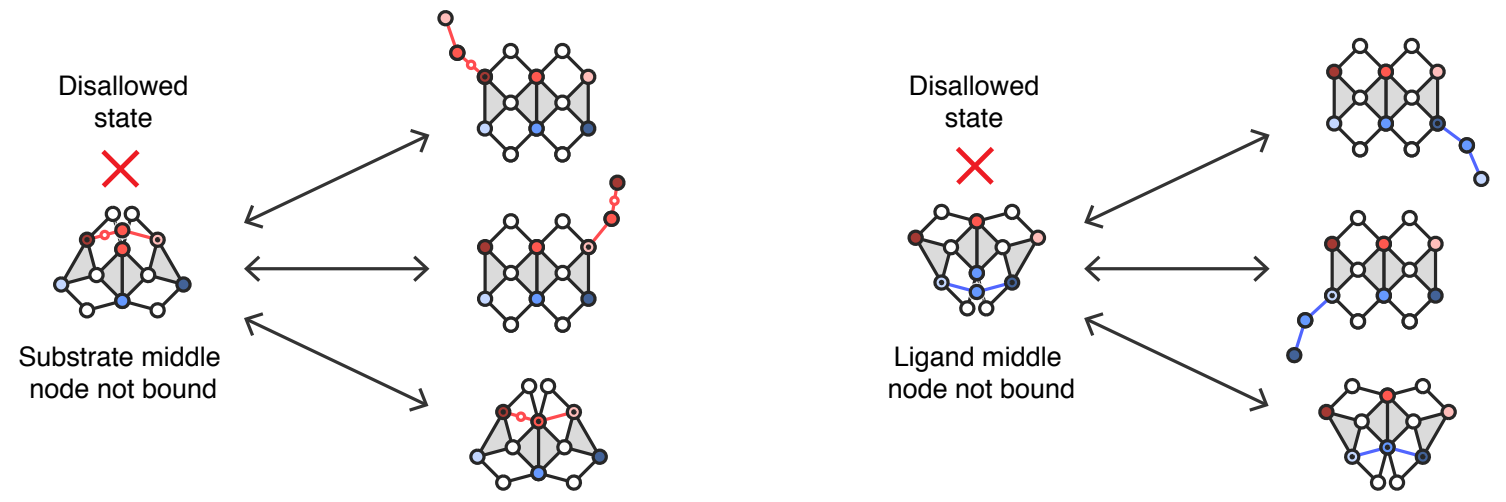

Fig. 12. Adjacency rule. The adjacency rule allows only adjacent bonds to form or dissociate between two interacting linkages. Consequently, two geometrically possible states are disallowed - one for the substrate (left), and one for the ligand (right) - in which the central node is not bound while the two outer nodes are bound.

\subsection{Comparison of species in myosin, DIV and TRV}

Fig. 13 compares the species, allosteric coupling, and catalytic reactions in myosin, DIV and TRV.
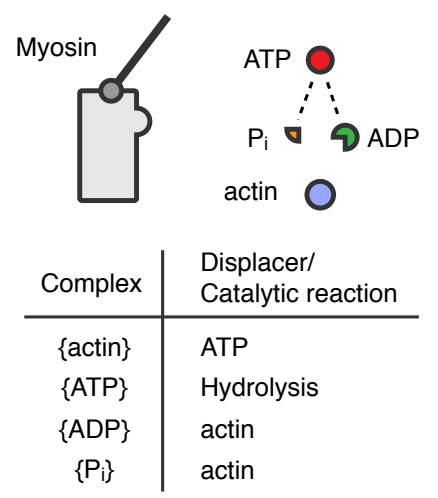
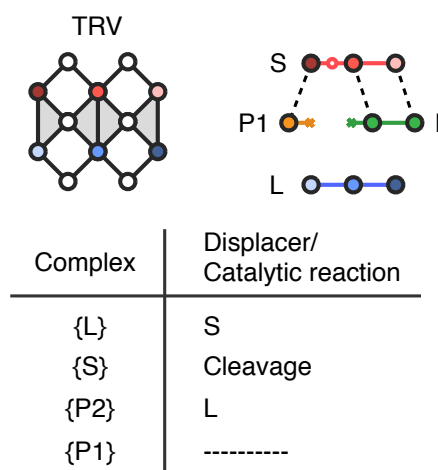
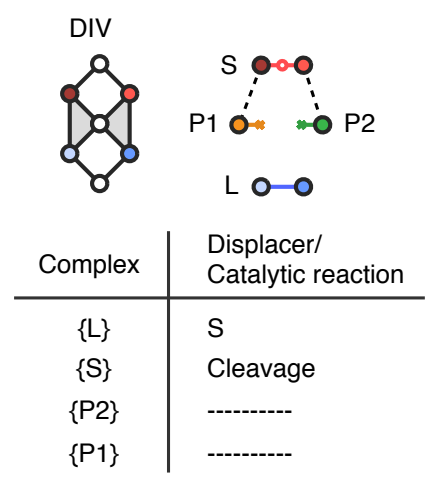

Fig. 13. Species, allosteric coupling, and catalytic reactions in myosin, DIV and TRV. Left, myosin. Center, TRV. Right, DIV. Species in each system are matched by row in the tables below.

\section{Simulating the myosin cycle with linkages}

The primary goal in running simulations was to show that the TRV model and allosteric mechanism proposed could generate myosin-like productive cycles. The secondary goal was to show that the DIV model, which lacks a mechanism for allosterically driven product release, would in some way fail to generate myosin-like productive cycles. I wanted to accomplish both goals using rates that had some basis in experimental data.

The simulations were set up as 'single-molecule simulations', in the sense that I only looked at the time evolution of a single enzyme as it reacted with high concentrations of substrate and 
ligand. Hence, simulation trajectories consisted only of states of the enzyme in complex with the four reactants (including the empty state, $\{\varnothing\}$ ). In all simulations, the concentrations of product were set to zero at the outset. Tables 1, 2 and 3 give the specific parameters and rates used to perform simulations. Simulations were done with the software StochPy [35], using the Next Reaction method.

I set the cleavage rate equal to the ligation rate to ensure that no directional bias was introduced by the catalysis interaction, and that cycling could be assessed as a function of reactant concentration, and the ability for the enzymes to release product. This choice was also meant to reflect the experimental observation inside an NTPase, hydrolysis can be a highly reversible process, with an equilibrium constant close to 1 [5, 36, 37]. Simulations are done in a regime where the rate of catalysis is comparable to the faster rates of dissociation (weakest node interactions). In this regime, random dissociations of P1 or P2 from the DIV enzyme, or P1 from the TRV enzyme, rectify the cleavage reaction.

Python routines were written to search simulation trajectories for sequences of states corresponding to productive and futile cycles, as defined in section 4.1 (Details to be included Methods).

Table 1: Categories of reaction rates

\begin{tabular}{ll} 
Parameter & Value \\
\hline Bimolecular rate constant, $k_{\mathrm{bi}}$ & $9 \times 10^{6} \mathrm{M}^{-1} \mathrm{~s}^{-1}$ \\
Intermolecular binding rates, $k_{m}$ 's & $k_{\mathrm{bi}} \times\left[\mathrm{m}^{\prime}\right]$ \\
Intramolecular binding rate, $k_{\text {uni }}$ & $1 \times 10^{6} \mathrm{~s}^{-1}$ \\
Dissociation rates, $k_{\mathrm{d}(\text { node) }}$ 's & varies with node \\
Catalysis, $k_{\text {cat }}$ & $100 \mathrm{~s}^{-1}$
\end{tabular}

Table 2: DIV dissociation rates

\begin{tabular}{lll} 
Rate & Reactants & Value \\
\hline$k_{\mathrm{d} 1}$ & $\mathrm{~S} / \mathrm{P} 1$ & $250 \mathrm{~s}^{-1}$ \\
$k_{\mathrm{d} 5}$ & $\mathrm{~S} / \mathrm{P} 2$ & $3 \mathrm{~s}^{-1}$ \\
$k_{\mathrm{d} 4}$ & $\mathrm{~L}$ & $200 \mathrm{~s}^{-1}$ \\
$k_{\mathrm{d} 2}$ & $\mathrm{~L}$ & $680 \mathrm{~s}^{-1}$
\end{tabular}

Table 3: TRV dissociation rates

\begin{tabular}{lll} 
Rate & Reactants & Value \\
\hline$k_{\mathrm{d} 4}$ & $\mathrm{~S} / \mathrm{P} 1$ & $250 \mathrm{~s}^{-1}$ \\
$k_{\mathrm{d} 0}$ & $\mathrm{~S} / \mathrm{P} 2$ & $3 \mathrm{~s}^{-1}$ \\
$k_{\mathrm{d} 7}$ & $\mathrm{~S} / \mathrm{P} 2$ & $680 \mathrm{~s}^{-1}$ \\
$k_{\mathrm{d} 11}$ & $\mathrm{~L}$ & $680 \mathrm{~s}^{-1}$ \\
$k_{\mathrm{d} 2}$ & $\mathrm{~L}$ & $200 \mathrm{~s}^{-1}$ \\
$k_{\mathrm{d} 8}$ & $\mathrm{~L}$ & $680 \mathrm{~s}^{-1}$
\end{tabular}




\subsection{The single-allosteric-unit (DIV) system}

In Fig. 14, the productive and futile cycles for the DIV system are arranged in a cube-like graph, as they were for myosin in Fig. 2, except that each vertex is expanded as need be to show a minimal sequence of steps required to satisfy the binding transitions. The productive and futile cycles are defined as follows:

Productive-cycle: Any of the three six-state cycles shown below. In all three sequences, P2 dissociates after the ligand binds:

$$
\begin{aligned}
& \text { 1. }\{\mathrm{L}\} \rightarrow\{\mathrm{S}, \mathrm{L}\} \rightarrow\{\mathrm{S}\} \rightarrow\{\mathrm{P} 1, \mathrm{P} 2\} \rightarrow\{\mathrm{L}, \mathrm{P} 1, \mathrm{P} 2\} \rightarrow\{\mathrm{L}, \mathrm{P} 2\} \rightarrow\{\mathrm{L}\} \\
& \text { 2. }\{\mathrm{L}\} \rightarrow\{\mathrm{S}, \mathrm{L}\} \rightarrow\{\mathrm{S}\} \rightarrow\{\mathrm{P} 1, \mathrm{P} 2\} \rightarrow\{\mathrm{P} 2\} \rightarrow\{\mathrm{L}, \mathrm{P} 2\} \rightarrow\{\mathrm{L}\} \\
& \text { 3. }\{\mathrm{L}\} \rightarrow\{\mathrm{S}, \mathrm{L}\} \rightarrow\{\mathrm{S}\} \rightarrow\{\mathrm{P} 1, \mathrm{P} 2\} \rightarrow\{\mathrm{L}, \mathrm{P} 1, \mathrm{P} 2\} \rightarrow\{\mathrm{L}, \mathrm{P} 1\} \rightarrow\{\mathrm{L}\}
\end{aligned}
$$

Idling: Takes place when cleavage happens before ligand is displaced by substrate, followed by the dissociation of product and the return to the ligand bound state $\{\mathrm{L}\}$.

Free-burn: Takes place when consecutive rounds of substrate cleavage happen without the involvement of the ligand. There are two pathways for the free-burn: (1) substrate binds to the empty state $(\{\varnothing\})$ after both products dissociate; or (2) substrate binds after one product has dissociated, but one remains.

Futile-reset: Takes place when ligand binds to the empty state $(\{\varnothing\})$, thus when P2 dissociates before the ligand binds.

Productive cycle 1 is the canonical myosin cycle. Productive cycle 2 is the target cycle for linkages. Productive cycle 3, in which P2 dissociates before P1, after the binding of ligand, is not shown in Fig. 14, but it satisfies the rule that ligand bind before P2 dissociates. Although states of the DIV system can be laid out to match the sequence in productive cycle 2, the DIV system does not actually have the required complexity to non-randomly go through productive 2, or any productive cycle. This is because the substrate and ligand processes are not reciprocally coupled in the DIV system: substrate allosterically displaces ligand, but ligand does not allosterically displace a product. The lack of reciprocal allosteric coupling greatly increases the systems probability of following the futile pathways. Thus, the DIV system is acting as a negative control for the concept that reciprocity is required for productive cycling. This is described in more detail below.

Allosteric coupling between the substrate and ligand processes is lost after cleavage of the substrate. Ligand is displaced by substrate in three steps (Fig. 14, three $\{\mathrm{S}, \mathrm{L}\}$ states). The direction of the reaction is governed by the tighter binding of substrate than ligand $\left(\varepsilon_{\text {substrate }}>\varepsilon_{\text {ligand }}>\varepsilon_{\mathrm{P} 2}\right)$. After displacement, the substrate is cleaved into P1 and P2. P1 and P2 are bound monovalently and thus there is no way to allosterically couple their dissociations to the binding of ligand, as defined geometrically for negative allosteric coupling in the linkage model: a monovalent association cannot create any rigidity in the structure of the enzyme that can be used as the basis for a geometric competition (see section 3.1 and Fig. 6). Consequently, P1 and P2 dissociate randomly according to the strengths of the nodes to which they are associated. If P2 dissociates after ligand binding the productive cycle is taken. If instead P2 dissociates before the ligand binds, or substrate binds 
before the ligand binds, the futile-reset (Fig. 14, purple path) or free-burn pathways (Fig. 14, orange path) are taken, respectively.

a

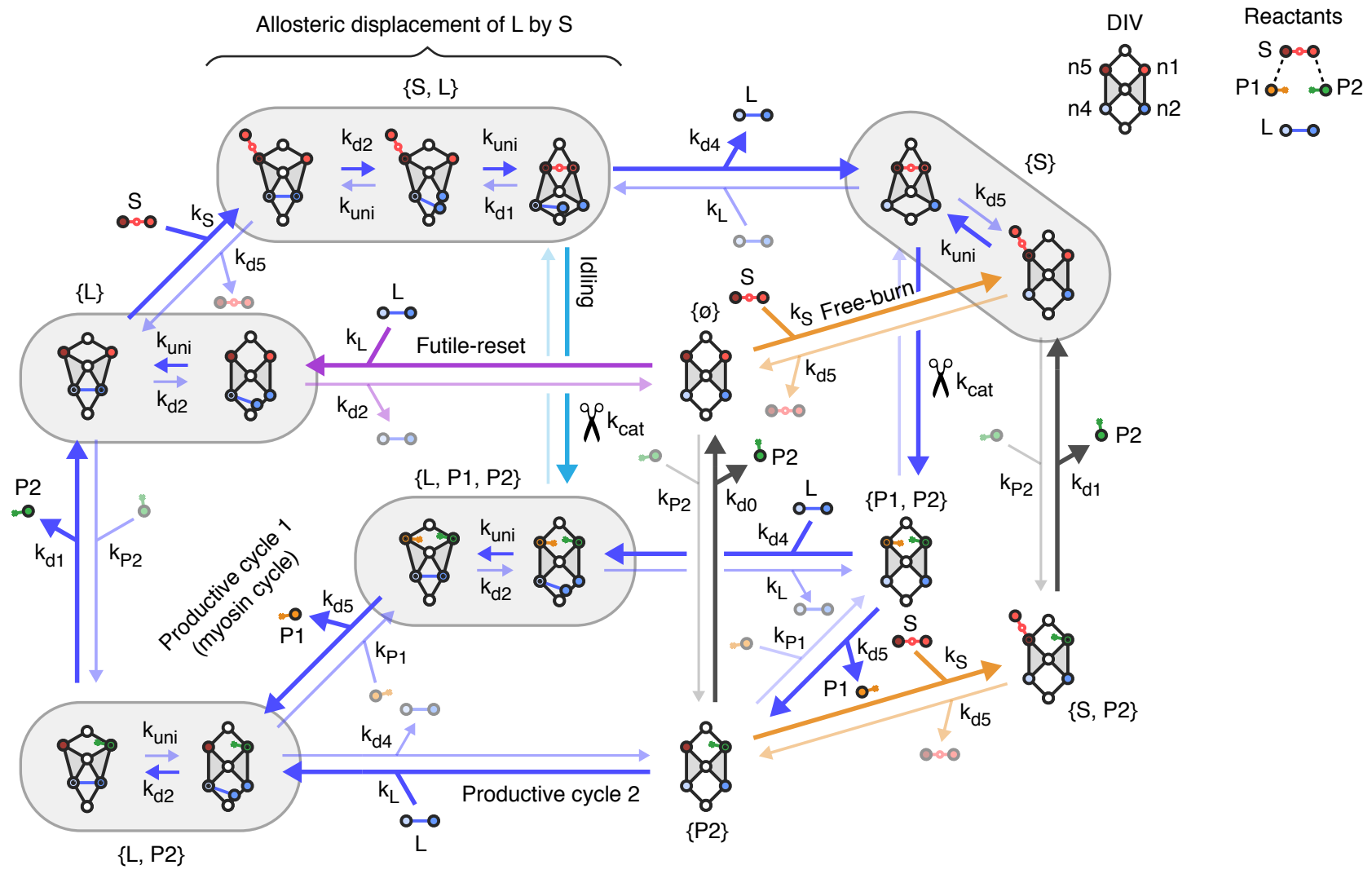

b Reaction cube

Productive cycle 2

Idling

Futile-reset (FR)

Free-burn (FB)
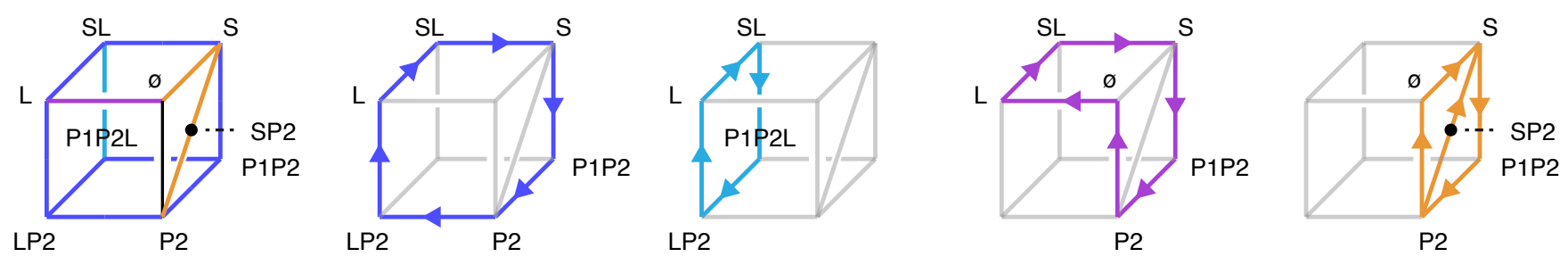

Fig. 14. Productive cycle and futile paths of the DIV system. a, Reaction cube for the DIV system in which each vertex is expanded as required to show a minimal path to complete a productive or futile cycle. There are nine vertices (grey bubbles surround isomers of the same composition), eight that make up the corners of the cube $(\{\mathrm{L}\},\{\mathrm{S}, \mathrm{L}\},\{\mathrm{S}\},\{\mathrm{P} 1, \mathrm{P} 2\},\{\mathrm{P} 2\},\{\mathrm{L}, \mathrm{P} 2\},\{\mathrm{L}, \mathrm{P} 1, \mathrm{P} 2\}$ and $\{\varnothing\})$, and one $(\{\mathrm{S}, \mathrm{P} 2\})$ that lies along the diagonal running from $\{\mathrm{P} 2\}$ to $\{\mathrm{S}\}$. A forward and reverse rate is given for each edge (forward path bold, reverse path translucent). While productive cycle 2 (the target) is displayed with the correct states, the DIV system does not have a reciprocal displacement system - S allosterically displaces $\mathrm{L}$, but both products dissociate randomly. Thus there is no mechanism to follow productive cycle 2 . The free-burn path is actually the favored path. b, Legend in which productive cycle 2 and the futile cycles are outlined separately on the cube. Vertices are labeled without brackets or commas between reactants (e.g. $\{\mathrm{S}, \mathrm{L}\}$ is $\mathrm{SL})$. 
The lack of control over path choice in the DIV system is shown in a plot of the simulation data (Fig. 15). Turnover rates for each of the four defined paths are reported as a function of substrate concentration. Ligand concentration was held constant (at $0.1 \mu \mathrm{M}$ ). The turnover rate is defined as the rate at which the product molecule P2 is created and then released by the enzyme (spurious binding of P2 followed by release is not counted as a turnover release). In addition to the four main paths, the plot also reports a total turnover rate (black line), which is a sum of the productive cycle rate and all possible futile paths (not all reported in this plot, to be included in supplemental section); and turnover rates for simulations done without ligand present in the system (No-ligand, grey line).
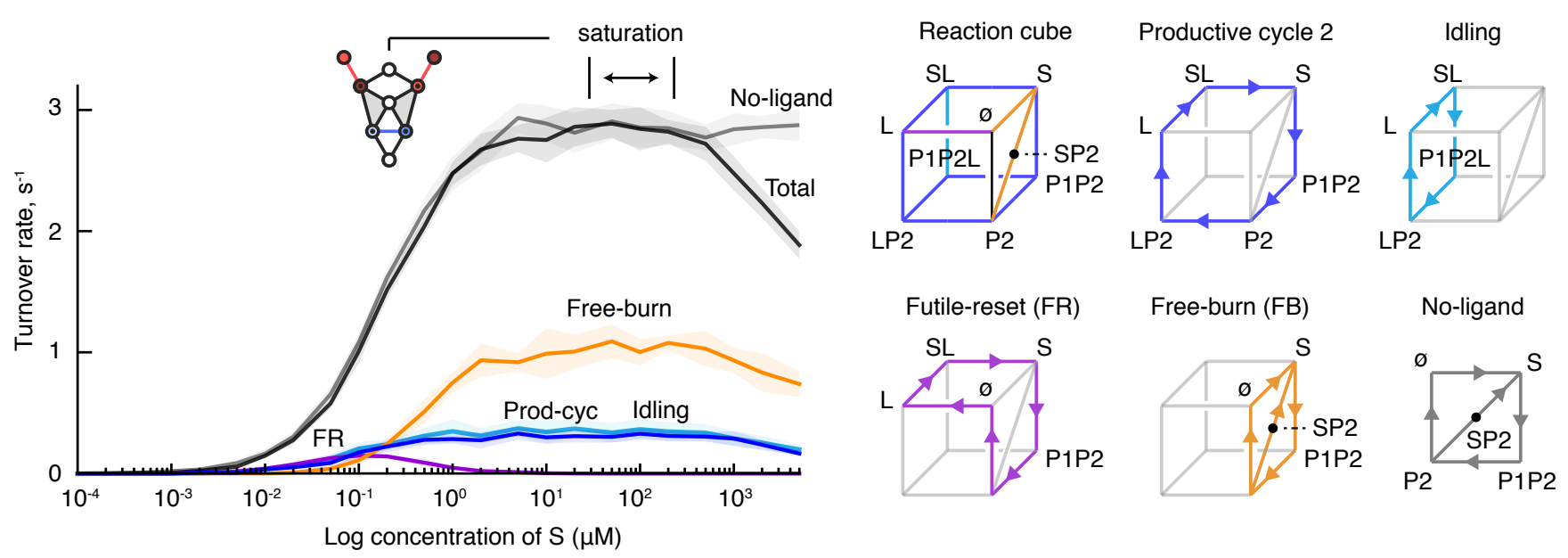

Fig. 15. Turnover rates of the different pathways in the DIV system. Left: Log scatter plot of turnover rates as a function of substrate concentration for the different cycles in the DIV system: productive (Prod-cyc, blue), futile-reset (FR, purple), free-burn (Free-burn, orange), and idling (Idling, turquoise). Additional curves are shown for the No-ligand condition (grey), in which simulations are run with substrate but no ligand, and Total condition (black), which is the total turnover rate with ligand including all productive and futile cycles. The DIV state $(\{\mathrm{S}, \mathrm{S}, \mathrm{L}\})$ that occurs at the point of substrate saturation is shown at top. Error bars are shown as colored shaded area around each scatter plot. Right: Legend in which productive cycle 2 and the futile cycles are outlined separately on the cube. Vertices are labeled without brackets or commas between reactants (e.g. $\{\mathrm{S}, \mathrm{L}\}$ is $\mathrm{SL}$ ). (Methods to be included).

Rather than the productive cycle, it is the free-burn path (the ligand-free process when ligand is present) that becomes the most likely path for the system to take as the concentration of substrate increases (Fig. 15, orange 'free-burn' curve). The productive path (blue 'prod-cyc' curve) is taken, at maximum, less than half the time the free-burn path is taken. As described above, there is no geometric control or allosteric control at $\{\mathrm{P} 2\}$ junction, where P2 is bound at a single node. Because of this, path choice at this junction and the $\{\varnothing\}$ junction is controlled only by the concentrations of ligand and substrate. Hence, as the concentration of substrate increases with a fixed ligand concentration, entry into the free-burn path increases much more than entry into the productive cycle.

The rate of idling tracks just above the rate of the productive cycle (Fig. 15, turquoise). It is not yet clear to me why my parameter assignments result in this behavior. But, this is an example of 
how the DIV system is less controlled than the TRV system, which is discussed in the next section.

Entry into the futile-reset path (Fig. 15, purple 'FR' curve) can be seen at lower concentrations of substrate (between 0.01 and $1 \mu \mathrm{M}$ ), where the probability that P2 is dissociates (leading to the empty state $\{\varnothing\}$ ) is comparable to the probability that substrate binds (leading to $\{\mathrm{P} 2, \mathrm{~S}\}$ ). As the concentration of substrate rises, entry into the free-burn path through the empty state begins to dominate over entry into the futile-reset path (Fig. 15, Prod-cyc network; black path connecting $\{\varnothing\}$ to $\{\mathrm{S}\})$. As a result, the turnover rate for the futile-reset path drops to near zero as the substrate concentration rises above $1 \mu \mathrm{M}$.

The ability for substrate concentration to so strongly effect path choice means that the system is not robust and has no inherent control. This is most clearly reflected by the fact that total turnover rate with ligand (Fig. 15, black 'Total' line) and turnover rate without ligand (grey 'No-ligand' line), track together until very high saturating concentrations of substrate. Before saturation the presence of the ligand is slightly inhibitory. The small lag is likely due to the time it takes for substrate to displace ligand, when it happens to be bound. When saturation is reached without ligand, the maximum turnover rate $\left(V_{\max }\right)$ is approximately the rate at which the system goes around the outside path in the No-ligand network, ignoring the substrate binding step (which is very relative to the other steps). This path includes catalysis, P1 dissociation and P2 dissociation:

$$
V_{\max } \approx\left(1 / k_{\mathrm{d} 4}+1 / k_{\mathrm{cat}}+1 / k_{\mathrm{d} 0}\right)^{-1}=2.8 \mathrm{~s}^{-1}
$$

P2 dissociation is rate limiting, because it is the slowest step $\left(k_{\mathrm{d} 0}=3 \mathrm{~s}^{-1}\right)$.

When ligand is present at saturating concentrations of substrate, the enzyme saturates with two substrates and one ligand (Fig. 15 plot, state labeled 'saturation'). In this state, the presence of ligand becomes strongly inhibitory, and catalysis is suppressed. Saturation begins to take place when the rate at which substrate binds begins to match, and then surpasses, its own node dissociation rates, and that of the ligand's. At this point, when ligand happens to be bound divalently, it is more likely for two substrates to bind in succession - one at each node - than it is for one substrate to bind and displace the ligand. Once this saturation complex can easily form, it becomes unlikely for substrate to bind divalently and be cleaved, and consequently the turnover rate drops as ligand stays bound for a long period of time (black 'Total' line, after $100 \mu \mathrm{M}$ ).

\subsection{The double-allosteric-unit (TRV) system}

In Fig. 16, states of the TRV system are arranged to match the productive path as it was shown for myosin in Fig 3, and the same three futile paths (futile-reset, free-burn and idling) are shown as they were defined for DIV. In contrast to the DIV system, the TRV system does possess the allosteric reciprocity between the substrate and ligand processes required to emulate the myosin system: substrate displaces ligand, and ligand displaces P2. The direction of the two reciprocal allosteric displacements are governed by the binding energies $(\varepsilon$ 's) of the reactants: substrate binds tighter than ligand, and ligand binds tighter than $\mathrm{P} 2\left(\varepsilon_{\text {substrate }}>\varepsilon_{\text {ligand }}>\varepsilon_{\mathrm{P} 2}\right)$.

Substrate is shown displacing the ligand in a minimal sequence of five steps along the productive cycle (Fig. 16, $\{\mathrm{S}, \mathrm{L}\}$ states). After the displacement of ligand by substrate, the cleaving of substrate into P1 and P2, and the random dissociation of P1, P2 remains bound to the enzyme at two nodes to form a stable complex $(\{\mathrm{P} 2\})$ at the junction where the productive cycle, free-burn path and futile-reset paths meet. 
Tight and divalent binding of P2 strongly inhibits diversions from the productive-cycle into the two futile pathways. Futile-resets - dissociations of P2 followed by ligand binding - are inhibited simply because P2 remains bound the enzyme. Transient and short-lived partial dissociations of P2 can take place, as depicted in the first transition along the futile-reset path, but intramolecular binding is so fast compared to dissociation, that P2 rebinds rather than dissociates from its second node (box 1, Fig. 16), which returns the system to the productive path.

The free-burn pathway is suppressed because P2 sterically blocks substrate from binding. This is accomplished by partitioning the substrate's binding energy so that the strongest node association is the central node (node 0), which is one of P2's nodes. The other two nodes are weaker, and thus stochastic dissociations are faster from them. This unequal partitioning makes it difficult for substrate to bind divalently and sterically displace P2 from the active site. When substrate does bind at node 4 after P1 dissociates, its relatively weak monovalent association with the enzyme makes it probable for it to dissociate before P2 dissociates from node 0 (box 2, Fig. 16). Because intramolecular binding is much faster than intermolecular binding, substrate will rarely get a chance to initiate a steric attack at nodes 0 or 7 , where P2 is bound divalently.

By contrast, the presence of P2 does not inhibit stable ligand binding, which favors continuation along the productive pathway at the junction. The geometry of the system, which allows for independent motion of the left and right allosteric units, means that the ligand can bind tightly and divalently while P2 is bound divalently. As depicted in the first two $\{\mathrm{L}, \mathrm{P} 2\}$ states after $\{\mathrm{P} 2\}$ along the productive cycle in Fig. 16, the ligand can in sequence bind monovalently and then divalently to the left side of the enzyme, while P2 remains divalently bound on the right side. In addition to the geometry of the enzyme, the rate assignments support divalent binding of the ligand because intramolecular binding to a node is much faster than dissociation from a node (Box 3, Fig. 16). Therefore, the ligand can easily return to the divalent state (2nd $\{\mathrm{L}, \mathrm{P} 2\}$ state) while it waits to complete the displacement of P2 from the enzyme. Thus, allosteric displacement of P2 by ligand in the productive cycle is favored over steric displacement of P2 by substrate along the futile-burn path. 
a

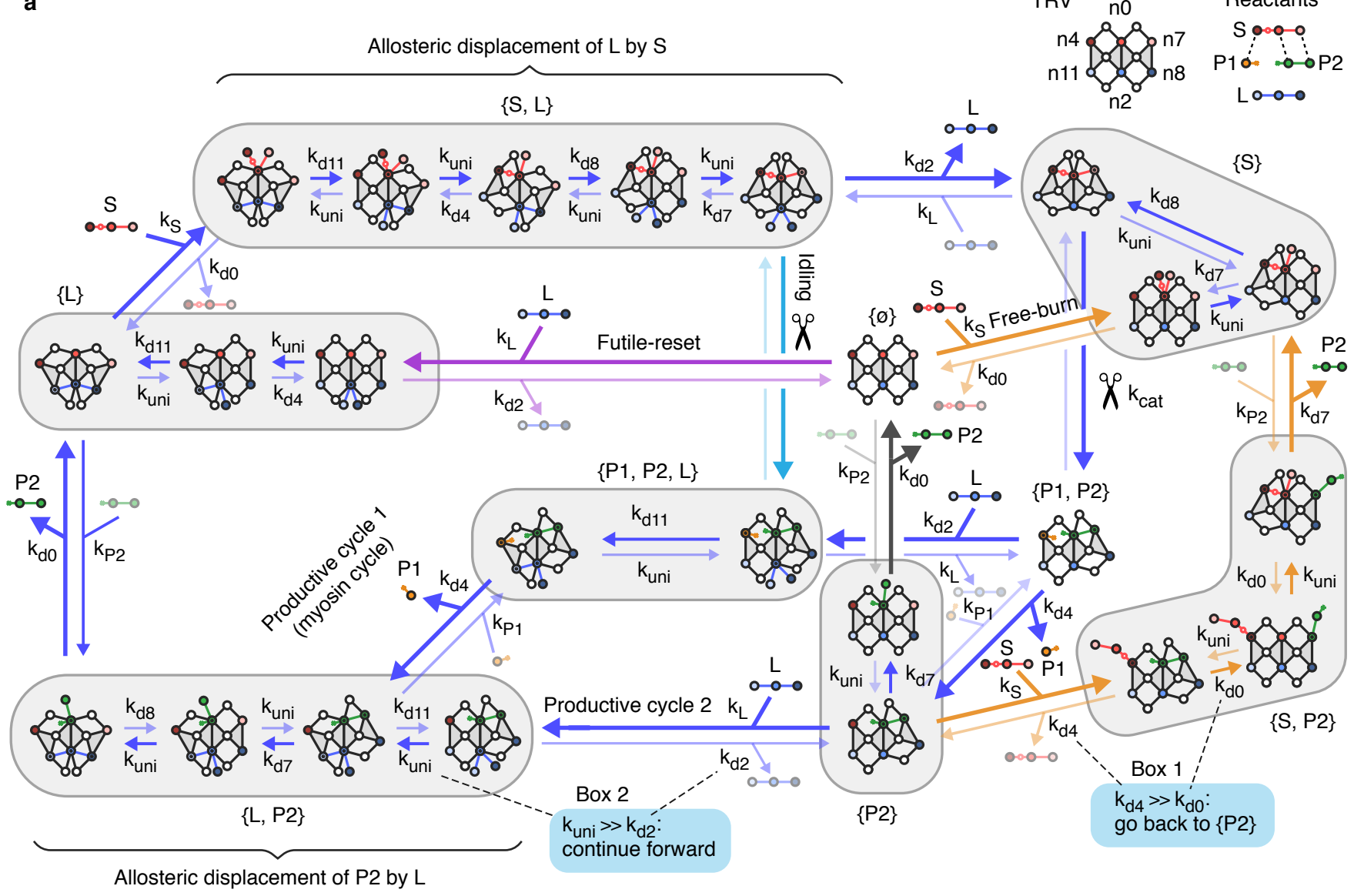

b

b Reaction cube

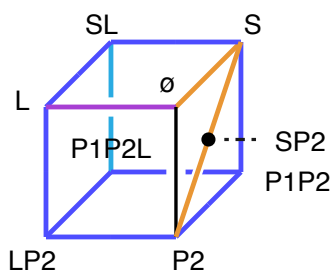

Productive cycle 2

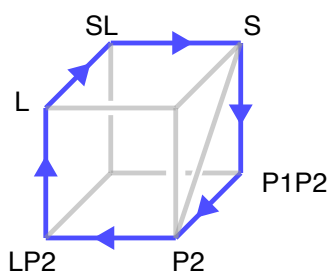

Idling

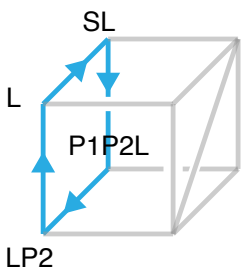

Futile-reset (FR)

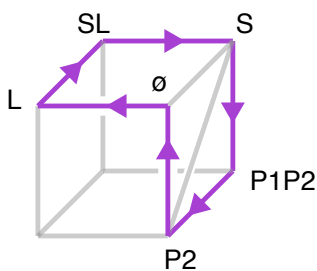

Free-burn (FB)

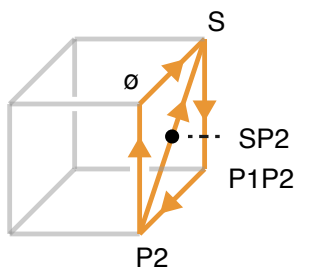

Fig. 16. Productive cycle and futile paths of the TRV system. a, Reaction cube for the TRV system in which each vertex is expanded as required to show a minimal path to complete a productive or futile cycle. There are nine vertices (grey bubbles surround isomers of the same composition), eight that make up the corners of the cube $(\{\mathrm{L}\},\{\mathrm{S}, \mathrm{L}\},\{\mathrm{S}\},\{\mathrm{P} 1, \mathrm{P} 2\},\{\mathrm{P} 2\},\{\mathrm{L}, \mathrm{P} 2\},\{\mathrm{L}, \mathrm{P} 1, \mathrm{P} 2\}$ and $\{\varnothing\})$, and one $(\{\mathrm{S}$, $\mathrm{P} 2\})$ that lies along the diagonal running from $\{\mathrm{P} 2\}$ to $\{\mathrm{S}\}$. A forward and reverse rate is given for each edge (forward path bold, reverse path translucent). The TRV system does have a reciprocal displacement system - S allosterically displaces L, and L displaces P2 (both displacement sequences are labeled with large curly braces). Thus, productive cycle 2 is the favored path. Continuation along productive cycle 2 at the $\{\mathrm{P} 2\}$ junction is supported by: (1) the divalent stability of P2 (no random dissociation to $\{\varnothing\}$ ); (2) the kinetic stability of $\{\mathrm{P} 2\}$, which can rebuff binding attempts by substrate (see Box 1); and (3) the vulnerability of P2 to displacement by the ligand, which can bind stably at two nodes while P2 is bound (see Box 2). b, Legend in which productive cycle 2 and the futile cycles are outlined separately on the cube. Vertices are labeled without brackets or commas between reactants (e.g. $\{\mathrm{S}, \mathrm{L}\}$ is $\mathrm{SL}$ ). 
In the idling path, like in the DIV system, the ligand remains bound during catalysis, the products dissociate, and the system returns to the starting configuration (Fig. 16, light blue 'Idling' path). The difference here is that P2 is allosterically displaced by ligand in the idling pathway. P1 is shown randomly dissociating before P2 is displaced by the ligand.
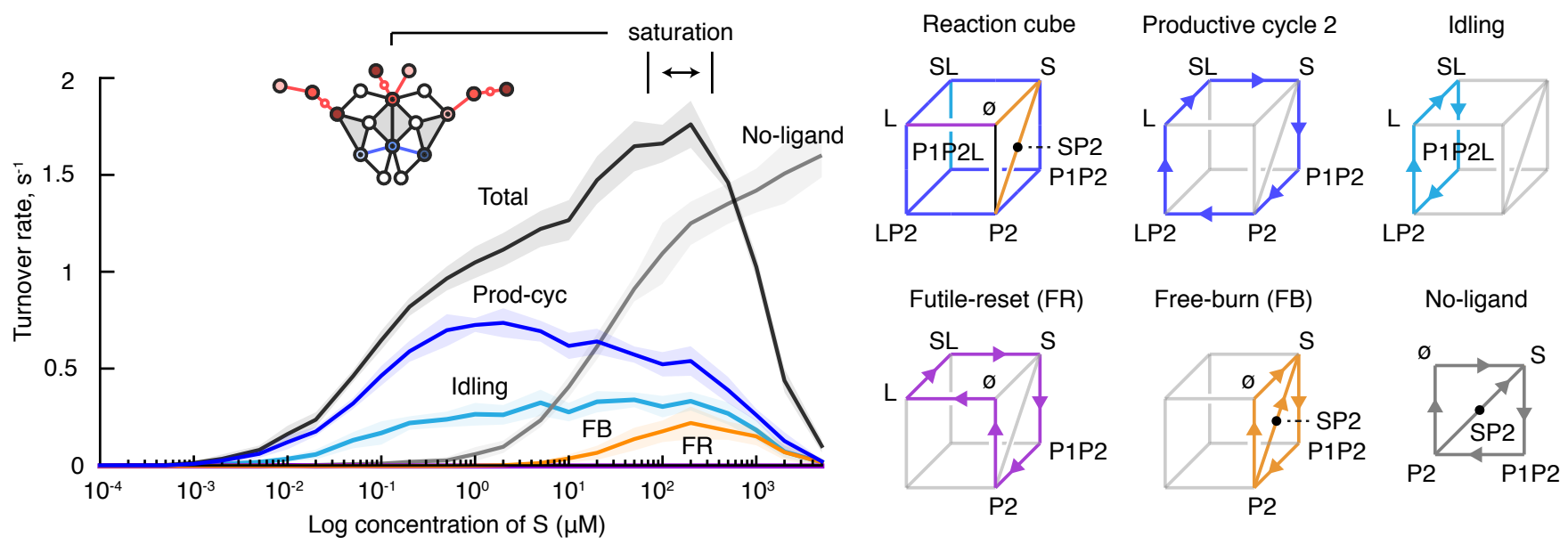

Fig. 17. Turnover rates of the different pathways in the TRV system. Left: Log scatter plot of turnover rates as a function of substrate concentration for the different cycles in the DIV system: productive (Prod-cyc, blue), futile-reset (FR, purple), free-burn (Free-burn, orange), and idling (Idling, turquoise). Additional curves are shown for the No-ligand condition (grey), in which simulations are run with substrate but no ligand, and Total condition (black), which is the total turnover rate with ligand including all productive and futile cycles. The TRV state $(\{\mathrm{S}, \mathrm{S}, \mathrm{S}, \mathrm{L}\})$ that occurs at the point of substrate saturation is shown at top. Error bars are shown as colored shaded area around each scatter plot. Right: Mininetworks showing the No-ligand condition pathways (top), and a reproduction of the normal condition with ligand that is shown in Fig. 16 (bottom)(Methods to be included).

The ability for the TRV system to favor the productive cycle over the futile paths is shown in a plot of the respective turnover rates for each path (Fig. 17). Over the full range of substrate concentrations tested, the productive cycle (blue line) remains the most probable. The turnover rate for the futile-reset path is zero over all $[\mathrm{S}]$ (purple line), reflecting that P2 does not randomly dissociate in TRV, as it does in DIV. The free-burn path (orange line), in which substrate sterically displaces P2, only becomes probable at very high concentrations of substrate, where the high incidence of substrate binding overcomes the likelihood that most binding events end with the substrate dissociating (Fig. 16, Box 2).

Idling occurs at roughly half the rate that the productive cycle occurs, until higher concentrations of substrate, where is the rate of idling increases but still does not surpass the productive cycle (Fig. 15 , turquoise vs. blue). In contrast to the DIV system, idling is more effectively suppressed in the TRV system, but again, the way in which parameter choice and design result in this outcome is not yet clear.

A comparison of the total turnover rate (Fig. 17, black line) and no-ligand rate (grey line), shows how the rate of catalysis with ligand remains faster than the rate without ligand for a large range of substrate concentrations, until they cross at $[\mathrm{S}]=500 \mu \mathrm{M}$. Unlike for DIV, saturation 
is not reached for the no-ligand condition, for the tested range of substrate concentrations. With ligand in solution (black line), saturation is reached around $[\mathrm{S}]=100 \mu \mathrm{M}$. Here, the slowdown is due to the same mechanism described for the DIV system - inhibition of catalysis by the presence of one ligand, and multiple substrates that inhibit each other from displacing the ligand (Fig. 17, TRV state labeled 'saturation').

\subsection{Comparison of ligand activation and efficiency in the two systems}

In Fig. 18, I compare the performance of the DIV and TRV systems in two ways. First, I compare the ability for ligand to stimulate enzymatic activity in each system, using a measure called the ligand-activation-factor $(A)$, which is defined as the ratio of the turnover rate with ligand $\left(v_{\mathrm{L}}\right)$, to the turnover rate without ligand $\left(v_{\text {noL }}\right)$ :

$$
A=\frac{v_{\mathrm{L}}}{v_{\mathrm{noL}}}
$$

Secondly, I compare efficiencies of the two systems. The efficiency $(E)$ is defined as the percentage of P2's made and released by the productive cycle $\left(v_{\mathrm{PC}}\right)$, with respect to the total turnover rate with ligand $\left(v_{\mathrm{L}}\right)$ :

$$
E=100 \times \frac{v_{\mathrm{PC}}}{v_{\mathrm{L}}}
$$

For the divalent system, ligand activation remains less than one, for all substrate concentrations (Fig. 18a, black dots). Values less than one reflect how the ligand inhibits rather than activates catalysis in DIV; first weakly, for concentrations of substrate below $500 \mu \mathrm{M}$, and then strongly, for [S] above $500 \mu \mathrm{M}$ (the transition from weak to strong inhibition can be seen in Fig. 15). By contrast, in the trivalent system, ligand activation rises to over $50 \mathrm{x}$ at $[\mathrm{S}]=0.1 \mu \mathrm{M}$ (Fig. 18a, blue dots). The ability of the ligand to act as a switch in the TRV system, by turning on enzymatic activity for a range of substrate concentrations, or likewise, for the enzyme to be relatively dormant in the presence of substrate alone, is consistent with the behavior of a myosin monomer, which displays strong actin-activated ATPase activity [38].

Fig. 18b compares the efficiencies of the two systems. TRV maintains an efficiency close to 70 percent for concentrations of substrate between $0.02 \mu \mathrm{M}$ and $1 \mu \mathrm{M}$. By contrast, the efficiency of the DIV system does not get over 25 percent. 

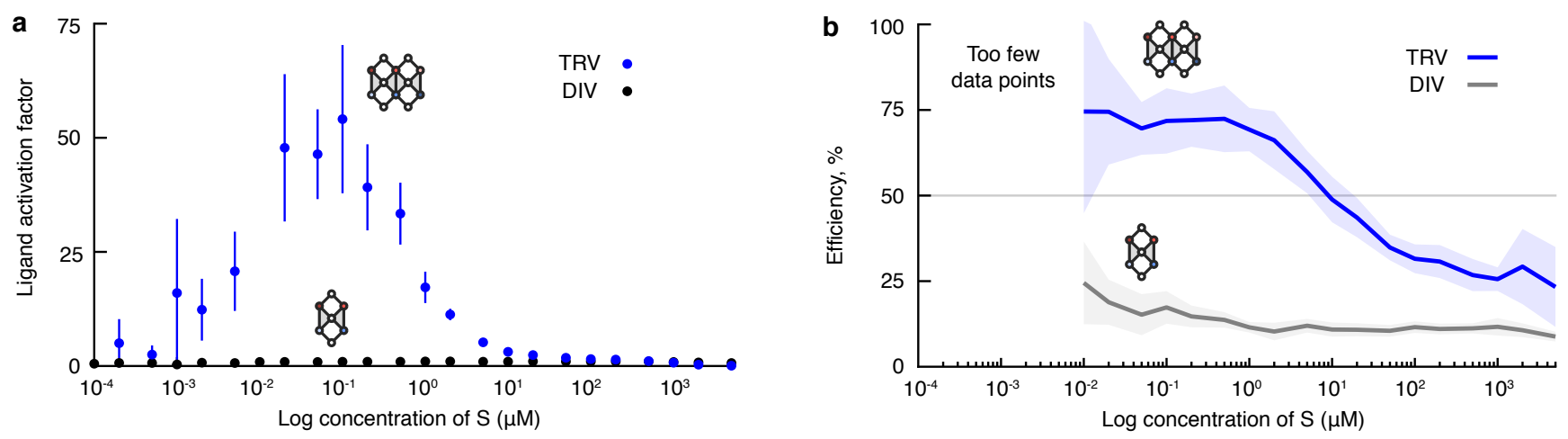

Fig. 18. Comparison of ligand activation and efficiency in DIV and TRV. Left, ligand activation in the two systems. Right, efficiency in the two systems. Error bars are shown as shaded area (Methods to be included).

\subsection{A closer look at the TRV trajectory}

Thirty seconds of a TRV simulation done at $100 \mathrm{nM}$ substrate and $100 \mathrm{nM}$ ligand is shown in Fig. 19. This particular segment was chosen because it shows a variety of behaviors. The trajectory shows how the substrate's time spent on the enzyme is minimal (Fig. 19a, red color bars). After rapidly displacing the ligand (where blue ends and red begins), the substrate is rapidly cleaved (where orange and green begin). For most of the trajectory, the enzyme is bound by P2 (green), ligand (blue) or both (overlap of green and blue). Each reactant can be seen as a bridge in the cycle: ligand bridges product to substrate; substrate bridges ligand to product; and product bridges substrate to ligand. The figure also shows how idling (grey bars) takes place within the time span of a productive cycle. The exception is when ligand dissociates after catalysis but before P2 dissociates, which is a rare event (Fig. 19a; Idling* between cycles 9 and 10). 

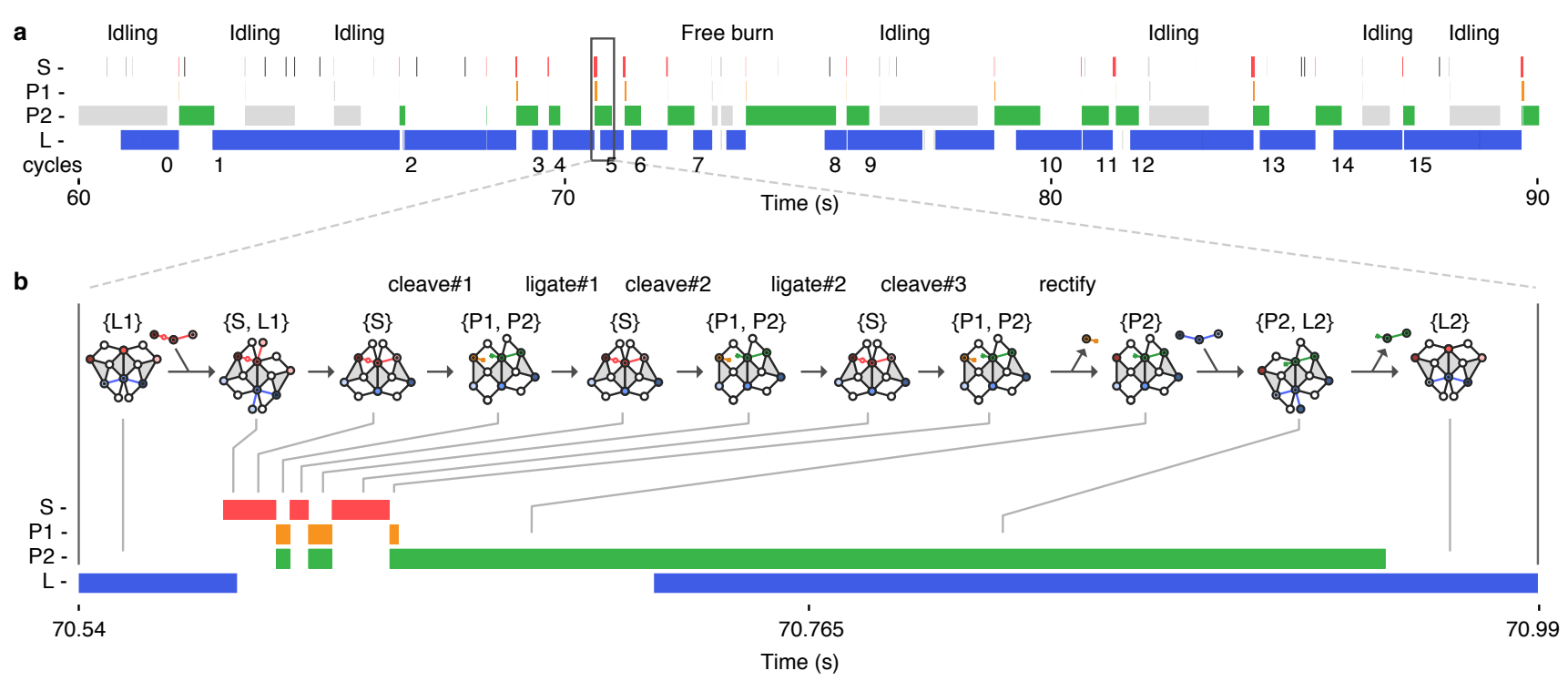

Fig. 19. Trajectory of TRV system. a, Thirty seconds of a ninety second trajectory done at $100 \mathrm{nM} \mathrm{S}$ and $100 \mathrm{nM} \mathrm{L}$. The time spend bound to the enzyme by each reactant is represented as a horizontal bar of color (S, red; P1, grey; P2, green; and L, blue). Grey bars show futile events, which are labeled at the top. The cycle count is shown at the bottom above the time, where each number after 0 marks the completion of a cycle with the enzyme bound to ligand only (state $\{\mathrm{L}\}$ ). b, A $70 \mathrm{x}$ zoom of the boxed section in $\mathbf{a}$ that demarcates 0.45 seconds of the trajectory. Each horizontal segment for which the composition of the enzyme changes is represented above by the most stable thermodynamically stable state of the complex. The consecutive cleavage and ligation events that take place are numbered above. The dissociation of P1 (labeled 'rectify') rectifies the last cleavage event (Methods to be included).

While the direction of the two allosteric displacements are designed to be controlled by binding energies, catalysis does not have directionality built into it. This reversibility can be seen in Fig. 19b, which shows a $70 \mathrm{x}$ zoomed in section of 0.45 seconds of the trajectory. The zoom shows how the catalysis reaction can toggle back and forth between cleavage and ligation, until P1 dissociates and rectifies the reaction. The release of P1 leaves P2 stably bound to the enzyme (state $\{\mathrm{P} 2\}$ ), allowing the displacement of P2 by ligand to proceed.

The whole sequence can be viewed as an energy cascade that is repeated each cycle (Fig. 20). In the energy cascade P2 binds the weakest, followed by ligand, followed by substrate. The cascading energy model borrows directly from the DNA strand displacement design [34, 39], in which a series of DNA strands that bind in sequence are often arranged as an energy cascade. With substrate bound, and the system at the bottom of the energy ladder, no other reactant can dislodge it from the enzyme. The cleavage reaction, and subsequent dissociation of P1 resets the cascade. 


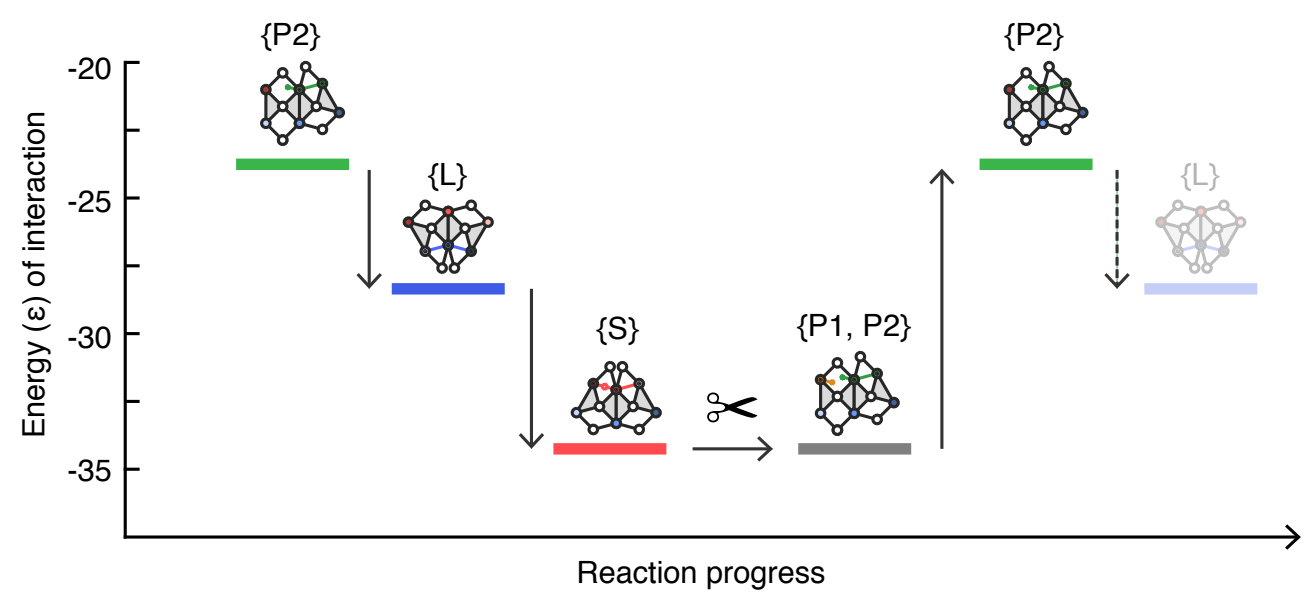

Fig. 20. Repeating cascade.

It is important to point out that in the modeling framework presented here, the internal energy of the enzyme complex remains the same before and after catalysis - the same bonds, with the same energies, are made by the substrate and P1 and P2 complexes. Hence, catalysis does not release energy or change the energy of the complex. In this model, catalysis is a gateway that changes the outcomes of individual dissociation events, and direction of the binding reactions. When the gate is closed (before cleavage), bound substrate may dissociate from node 4 with rate $250 \mathrm{~s}^{-1}$ and then rebind. By contrast, when the gate is open (after cleavage), P1 will dissociate from node 4 with the same rate, but diffuse away. Likewise, when the gate is closed, ligand will be rebuffed by the tighter binding substrate, but will displace P2 when the gate is open.

\subsection{A comparison of DIV, TRV and myosin}

The model presented here shows how a system linkages can be used emulate the ordered cycling of myosin given that: (1) one of the product bound states is kinetically stable with respect to steric displacement by fuel; and (2) that same product is allosterically displaced by a ligand (i.e. is kinetically unstable with respect to allosteric displacement by a ligand) (Fig. 21). To demonstrate this, the DIV system acts as a negative control, as it contains neither mechanism, and thus ends up being dominated by the free-burn cycle, which is the ligand-free process (Fig. 21, left). The TRV system, which contains both forms of control, is dominated by productive cycle 2 (Fig. 21, center). Myosin, which contains an even more stable product bound state, with two stages of allosteric displacement, is dominated by productive cycle 1 (Fig. 21, right). 

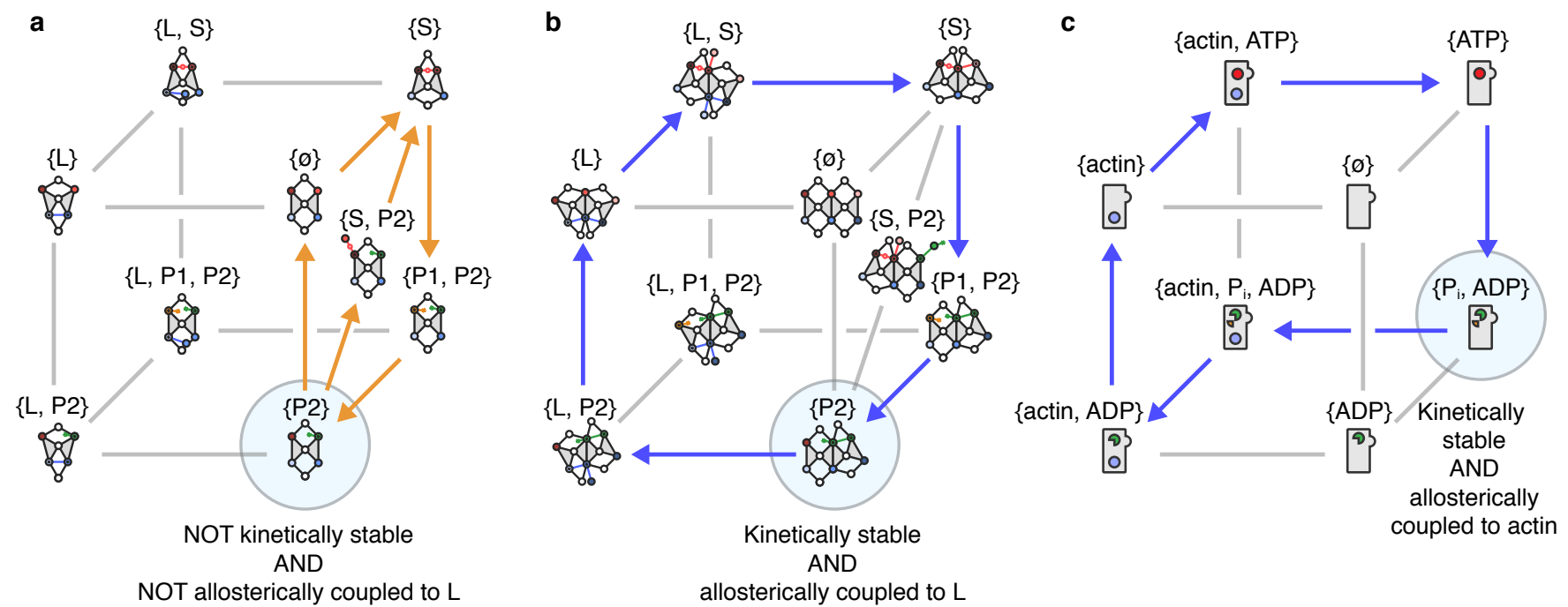

Fig. 21. Dominant cycles of DIV, TRV and myosin. a, DIV reaction cube minimized to nine states, with one state chosen to represent a set of isomeric states. DIV is dominated by the free-burn cycle (orange), which is the ligand-free process. State $\{\mathrm{P} 2\}$ (light blue circle) is not stable with respect to attack by substrate and random dissociation, and dissociation of P2 is not allosterically coupled to ligand. Consequently P2 easily dissociates before or while substrate is bound, resulting is the free-burn path through $\{\varnothing\}$ or $\{\mathrm{S}, \mathrm{P} 2\}$, respectively. $\mathbf{b}$, TRV reaction cube minimized to nine states, with one state chosen to represent a set of isomeric states. TRV is dominated by productive cycle 2 (blue path), a reciprocal cycle. State $\{\mathrm{P} 2\}$ (light blue circle) is stable with respect to attack by substrate and random dissociation, and dissociation of P2 is allosterically coupled to ligand binding. Consequently, TRV contains a mechanism to go through state $\{\mathrm{L}, \mathrm{P} 2\}$ and productive cycle 2, and avoid the non-reciprocal futile cycles through state $\{\varnothing\}$ and $\{\mathrm{S}, \mathrm{P} 2\}$. c, Myosin reaction cube showing its path through productive cycle 1 (the canonical myosin cycle). State $\{\mathrm{Pi}, \mathrm{ADP}\}$ (light blue circle) is kinetically stable with respect to ATP binding, and the dissociations of both $\mathrm{Pi}$ and $\mathrm{ADP}$ are allosterically coupled to actin binding. Consequently, the myosin system travels a path through state $\{$ actin, ADP, Pi $\}$, which even more so than productive cycle 2 , avoids the futile pathways.

The different levels of control and tight coupling in each system is reflected in a comparison of their ligand activation factors - the degree to which ligand (actin) increases catalytic activity (in myosin this is called actin associated ATPase activity). In DIV, ligand slightly inhibits catalytic activity. In TRV, ligand increased catalytic activity 50-fold. In myosin, actin increases catalytic activity over 700-fold [38]. Presumably, a linkage system with two stages of allosteric product displacement would further increase the ligand activation factor.

Below I explicitly discuss how clockwise directionality is established in the productive cycle of TRV by answering four questions about how the forward versus reverse direction is favored at each stage of the cycle. I use the discussion to compare TRV's behavior to myosin. The four questions reference the productive cycles of TRV and myosin shown in Fig. 21:

1. What mechanism drives substrate binding vs P2 binding at the ligand bound state $(\{\mathrm{L}\}(\{$ actin $\}$ in myosin)?

Substrate binding is driven by mass action, or a high concentration of substrate relative to P2. 
2. What does cleavage accomplish at the substrate bound state $(\{S\})$ that allows ligand to easily displace P2, but conversely ligation undoes at $\{\mathrm{P} 1, \mathrm{P} 2\}$ that allows ligand to be easily displaced by substrate?

The cleavage reaction creates a more flexible complex when it divides the relatively rigid substrate complex into the P1 and P2 complex (Fig. 21; $\{\mathrm{S}\}$ vs $\{\mathrm{P} 1, \mathrm{P} 2\}$ ). This change transforms the substrate into two separate P1 and P2 thermodynamic systems housed within one enzyme complex. Hence, the cleavage reaction acts as a thermodynamic and geometric partitioning reaction. The partitioning places the binding energy of P2 lower than that of the ligand, which allows the ligand to allosterically displace P2 from TRV. Conversely, ligation reverses the partitioning by transforming P1 and P2 into substrate, a single thermodynamic system. Ligation thus places the binding energy of the substrate higher than that of the ligand, allowing the substrate to rebuff any attempts by ligand to displace it from TRV.

In myosin, hydrolysis may also create a more flexible complex [28]. The reaction enables actin to displace both Pi and ADP from myosin, however, the nature of the allosteric displacement reactions in myosin is not well understood. Likewise, ligation of ADP and Pi into ATP should in principle be able to drive myosin off actin. Although, to my knowledge, this is not a phenomenon that has been captured or studied in myosin.

3. What mechanism enables ligand to bind rather than substrate at the P2 bound state $(\{\mathrm{P} 2\})$ ?

The binding of ligand is biased over substrate because P2 sterically blocks the substrate from binding, while allosterically allowing ligand to bind. This preference for ligand is an example of a kinetic control mechanism, because substrate binds more tightly to the enzyme than ligand, thus the system is asking a thermodynamically weaker state (ligand bound) to happen before a stronger state (substrate bound), though both substrate and ligand compete for binding. As seen in the plot of simulation data, ligand binding (the productive cycle) takes place over substrate binding (free-burn pathway) even when the chemical potential of substrate is much higher than that of the ligand (Fig. 17).

In myosin, the myosin-ADP-Pi complex is kinetically stable, so there is no place for ATP to bind, which prevents nucleotide exchange until actin binds. The kinetic stability of the myosin-ADP-Pi complex is the mechanism by which myosin avoids the futile cycles (i.e. free-burn and futile-reset) defined here.

4. What mechanism allows the displacement reactions to complete in the desired counterclockwise direction?

The direction of the two allosteric displacement reactions are driven by binding energies $(\varepsilon)$, where the stronger binding species is designed to displace the weaker binding species (Fig. 21; allosterically "active" states $\{\mathrm{S}, \mathrm{L}\}$ and $\{\mathrm{L}, \mathrm{P} 2\})$. The overall cycle is organized as an energy cascade, where $\varepsilon_{\mathrm{S}}>\varepsilon_{\mathrm{L}}>\varepsilon_{\mathrm{P} 2}$. In myosin, the same energy cascade may exist, considering that ATP binds more tightly to myosin than actin [40], and actin binds more tightly than Pi and ADP. However, in myosin, the mechanism by which an allosteric displacement works is not clear. Here, the mechanism of allosteric displacement is clearly described. The combination of an allosteric displacement mechanism, a catalytic mechanism that resets an energy cascade, and mass action, allows continuous bidirectional allosteric switching to take place in the TRV system. 


\section{Mechanical cycle of TRV}

In this section I describe the mechanical cycle of the TRV system and show how two units of TRV can be combined to make a dimeric walker that walks on a ligand "track".

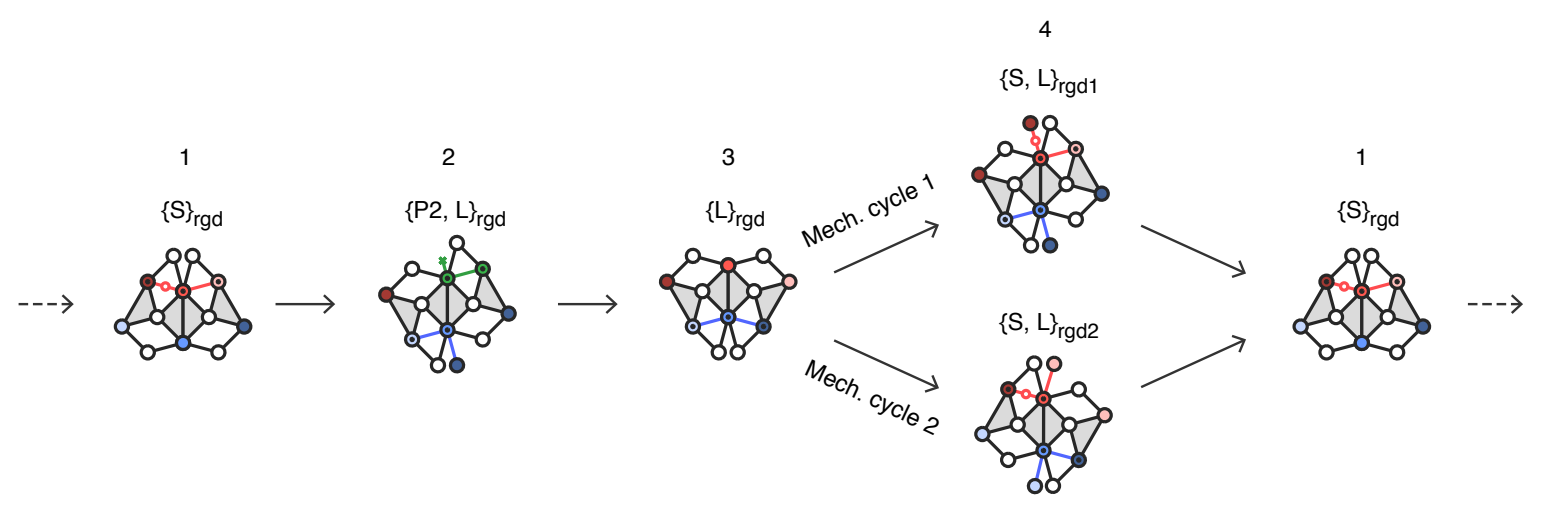

Fig. 22. Mechanical states of TRV. The five mechanical states of the system, or states that are completely rigid when bound, are shown in the order in which they occur within a productive cycle, starting with the enzyme bound to substrate. They are: $\{\mathrm{S}\}_{\mathrm{rgd}},\{\mathrm{P} 2, \mathrm{~L}\}_{\mathrm{rgd}},\{\mathrm{L}\}_{\mathrm{rgd}}$, and $\{\mathrm{S}, \mathrm{L}\}_{\mathrm{rgd} 1}$ and $\left(\{\mathrm{S}, \mathrm{L}\}_{\mathrm{rgd} 2}\right.$. The subscript 'rgd' denotes the state is a 'rigid' state. The substrate-ligand bound set of states $\{\mathrm{S}, \mathrm{L}\}$ has two rigid states denoted by 'rgd1' and 'rgd2'. Consequently, there are two mechanical paths through a productive chemical cycle, mechanical cycle 1 and mechanical cycle 2.

To associate the TRV chemical cycle with a mechanical cycle, I first define the meaning of mechanical states in the linkage model. Mechanical states of the enzyme are defined as completely rigid states of the enzymes. The system has five well-defined mechanical states: $\{\mathrm{L}\}_{\mathrm{rgd}}$, ligand bound trivalently; $\{\mathrm{P} 2, \mathrm{~L}\}_{\mathrm{rgd}}$, ligand and P2 bound divalently; $\{\mathrm{S}\}_{\mathrm{rgd}}$, substrate bound trivalently; and $\{\mathrm{S}, \mathrm{L}\}_{\mathrm{rgd} 1}$ and $\{\mathrm{S}, \mathrm{L}\}_{\mathrm{rgd} 2}$, two states in which substrate and ligand are bound divalently. The degeneracy in the $\{\mathrm{S}, \mathrm{L}\}$ states leads to two different mechanical paths that the TRV system can go through to complete a productive cycle. These two paths are shown in Fig. 22, with the cycles beginning in the substrate bound state. The two paths have the same chemical cycle, because the same reactants are bound at each stage, but different conformational cycles, because two different conformations can be adopted in state $\{\mathrm{S}, \mathrm{L}\}$. 

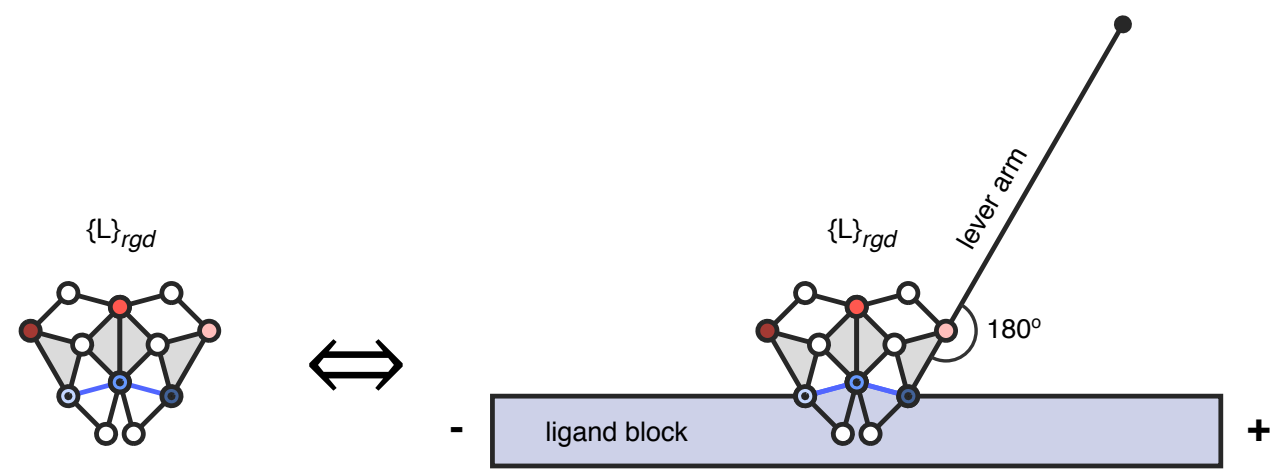

Fig. 23. TRV on a ligand track. The ligand molecule can be turned into block unit akin to an actin monomer and a polarized track can be formed to which TRV binds. A lever arm emerges at 180 degrees from the right edge of the right allosteric unit. When TRV is fully bound to the track the lever arm points towards the ' + ' direction of the track. The bound track state, which is rigid, is equivalent to the bound state on the left that is inscribed with the three-link. swimmer.

To relate the solution phase mechanical cycles to myosin-like track motion, I convert the ligand to a ligand block, and add a lever arm to the enzyme that emerges at a 180 degree angle from the edge of the right-hand allosteric unit (Fig. 23). Like actin, which is polar, the ligand block has a plus end ('+'), which is the intended direction of motion, and a minus end ('-'). In the reference frame of the ligand block, the motion of the lever arm projects the conformational changes of the enzyme as linear translations along the ligand block (Fig. 24). In both mechanical cycles, the enzyme binds to the track in state $\{\mathrm{P} 2, \mathrm{~L}\}_{\mathrm{rgd}}$, with its lever arm pointing towards the minus end of the track and then swings its lever arm towards the plus end after trivalently binding to the track (Fig. 24, steps 1-3). This step creates a forward bias in the 'landing phase' of the cycle, forward bias because the lever is pointing toward the plus end. The difference between mechanical cycle 1 and mechanical cycle 2 can be clearly seen in the next phase, the pick-up phase, when substrate binds and displaces the head from the track. In state $\{\mathrm{S}, \mathrm{L}\}_{\mathrm{rgd} 1}$ (Fig. 24, top path), the lever arm remains pointing towards the plus end, and retains the forward bias introduced during the landing phase. Conversely, in state $\{\mathrm{S}, \mathrm{L}\}_{\mathrm{rgd} 2}$ (Fig. 24, bottom path), the lever arm reverts to pointing towards the minus end, erasing the forward bias introduced during the landing phase.

The general question is, how can the monomer mechanical cycle, with two different pathways, be used to construct a forward walking dimer? There are two ways a dimeric motor can achieve forward motion along a polymer, a pick-up bias and a landing bias [41, 42]. A pick-up bias means that the rear head is more likely to be displaced from the track by substrate binding, and therefore even without a bias for landing in the forward position, the dimer can move in the forward direction. A pick-up bias also confers processivity to a walker, meaning it increases the probability that one leg remains bound to the track during the walking cycle. A landing bias means that a newly displaced and tethered head is more likely to bind in the forward position, thus even if there is no pick-up bias, forward motion of the dimer is possible. Certain dimeric myosins contains both a pick-up and landing-bias, which makes them both unidirectional and processive.

Pick-up and landing biases can be thought of as a phasing problem, where ideally each head needs to be in different parts of the mechanical and chemical phase of the cycle to achieve both 
forward motion and remain attached to the track. In myosin it is known that tension between the heads keeps the two heads out of phase chemically and mechanically. Here I show how tension between two TRV heads can be used to control the relative phases of the heads and mechanical pathway taken, resulting in a walker with both a pick-up and landing bias, and an operational cycle similar to dimeric myosin.

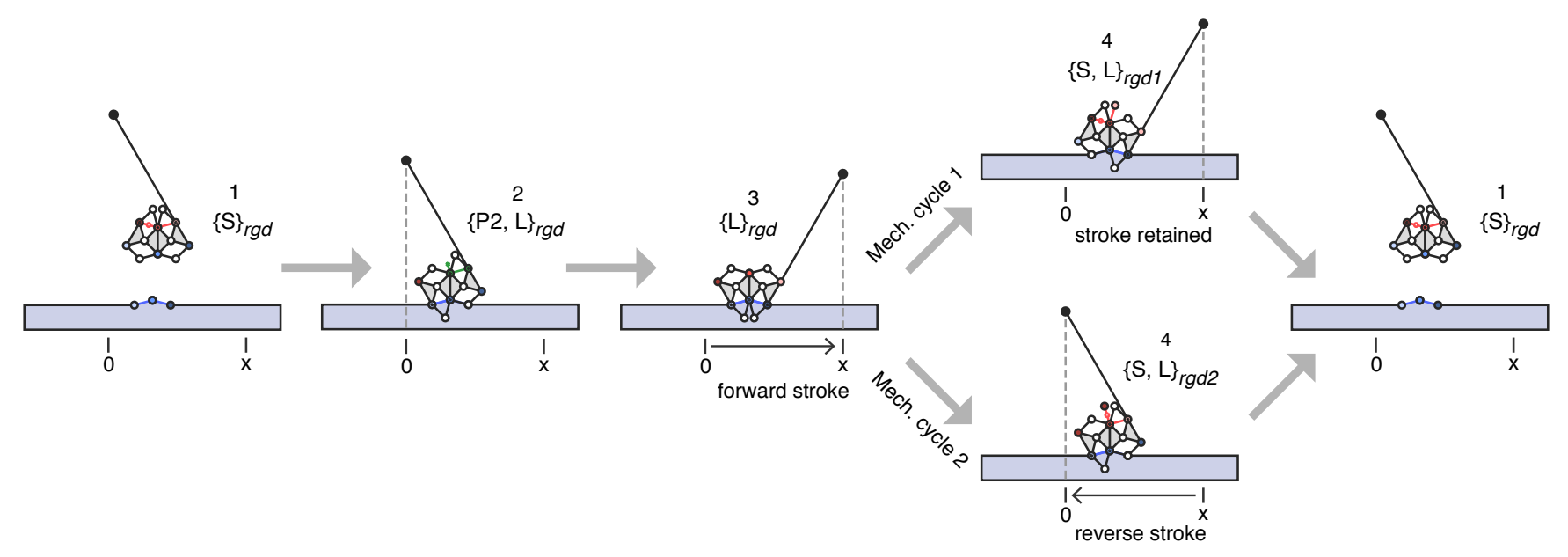

Fig. 24. The two mechanical cycles on a ligand block. The five mechanical states are shown on the ligand block, where mechanical cycle 1 follows the top path and mechanical cycle 2 follows the bottom path. When the enzyme first binds the track in step 2, and in state $\{\mathrm{P} 2, \mathrm{~L}\}_{\mathrm{rgd}}$, the lever arm points towards the minus end of the track and lines up with reference point 0 . Upon transitioning to state $\{\mathrm{L}\}_{\mathrm{rgd}}$ and fully binding to the track in step 3, the lever arm strokes forward to the plus end of the ligand block and lines up with reference point $\mathrm{x}$. If mechanical cycle 1 is followed, and the enzyme transitions to state $\{\mathrm{S}, \mathrm{L}\}_{\mathrm{rgd} 1}$, the lever arm remains lined up with point x. Conversely, if mechanical cycle 2 is followed, and the enzyme transitions to state $\{\mathrm{S}, \mathrm{L}\}_{\mathrm{rgd} 2}$, the lever arm strokes back to the minus side and lines with point 0 again. In either case, after the enzyme is displaced from the ligand block, the enzyme returns to state and the enzyme transitions to state $\{\mathrm{S}\}_{\mathrm{rgd}}$.

In the TRV walker design, a short tether connects two heads together such that tension constrains the motion of the heads (Fig. 25). The tension has the dual role of creating a pick-up bias for the rear head, and biasing the pick-up event to take place through mechanical cycle 1 . The pick-up bias for the rear head is created by rearward tension on the front head which prevents the front head from fully rotating clockwise and fully binding to the track, while the rear head is fully bound to the track (Fig. 25, lower right). The tension inhibits P2 from being displaced from the front head by track binding, and so the head is able to hold on to P2 in state $\{\mathrm{P} 2, \mathrm{~L}\}_{\mathrm{rgd}}$. The presence of P2 on the front head inhibits substrate binding to the front head, and biases substrate to displace the rear head. Bias for pick-up of the rear head through mechanical cycle 1 is created by forward tension on the rear head (Fig. 25, lower left). Forward tension inhibits the counterclockwise rotation of the rear head required for substrate to bind divalently the left allosteric unit, and displace the head from the track through mechanical cycle 2. Here we see how tension is a form of allosteric control between two enzymatic units that contains directionality or a vector component. 


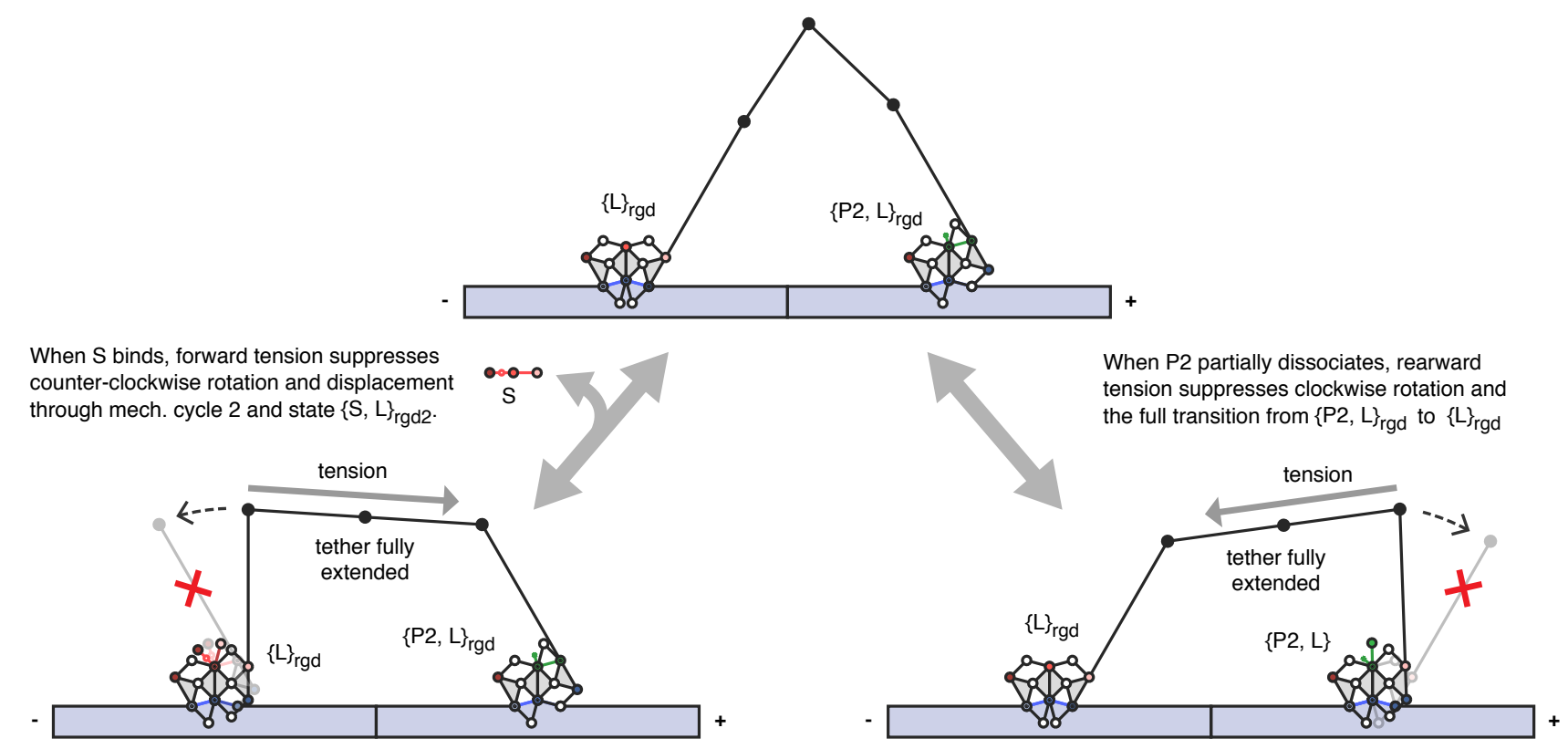

Fig. 25. Tension mediated restriction of motion in the front and rear head of the ideal dimer model. Top, the ideal dimer model in a stable configuration expected right after the front head binds while still holding on to P2. The shorter tether in this model allows the two heads remain out of phase chemically and mechanically when they are bound, with the rear head in () and the front head in (). The tether strongly biases the rear head to dissociate from the track through mechanical cycle 1 after S binds. Bottom right, if P2 dissociates from one of its nodes, flexibility in the tether allows some clockwise rotation, but not enough for the front head to bind trivalently to the track. Thus rearward tension inhibits P2 from dissociating from the front head and lever arm from stroking toward the plus end of the track. Bottom left, when the rear head dissociates from a track node while $\mathrm{S}$ is bound, tension prevents the head from rotating counterclockwise enough for $\mathrm{S}$ to displace the head from the track through mechanical cycle 2. Since mechanical cycle 1 does not require counterclockwise rotation of the head, mechanical cycle 1 is strongly biased over mechanical cycle 2 for pick-up of the rear head. 
1. Starting configuration that prevents read head from going through mech cyc. 2 and front head from fully binding to the track.

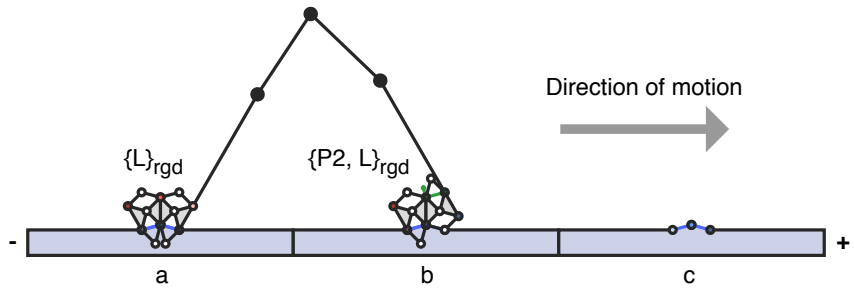

3. Tethered head is in state $\{\mathrm{P} 2\}$ after catalysis and the dissociation of $\mathrm{P} 1$. The front head remains $\{\mathrm{P} 2, \mathrm{~L}\}_{\mathrm{rgd}}$.

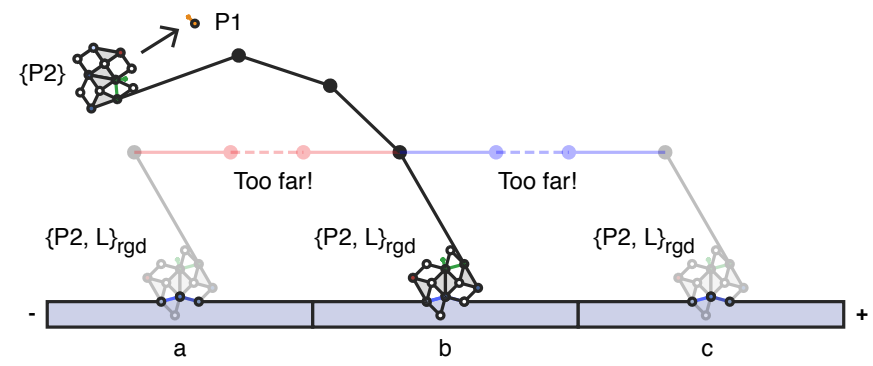

2. $S$ binds to rear head and the head transitions from $\{L\}_{\text {rgd }}$ to $\{S, L\}_{\text {rgd2 }}$.

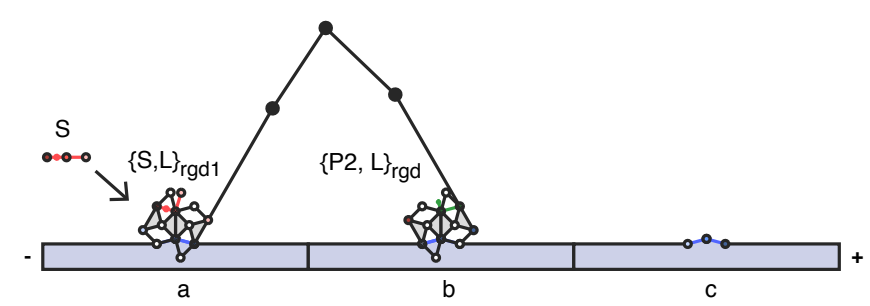

4. Bound head is in state $\{L\}_{\text {rgd }}$ after the lever arm stroke takes and $\mathrm{P} 2$ dissociates.

5. Tethered head binds at site $\mathrm{c}$ in state $\{\mathrm{P} 2, \mathrm{~L}\}_{\mathrm{rgd}}$ and becomes lead head.
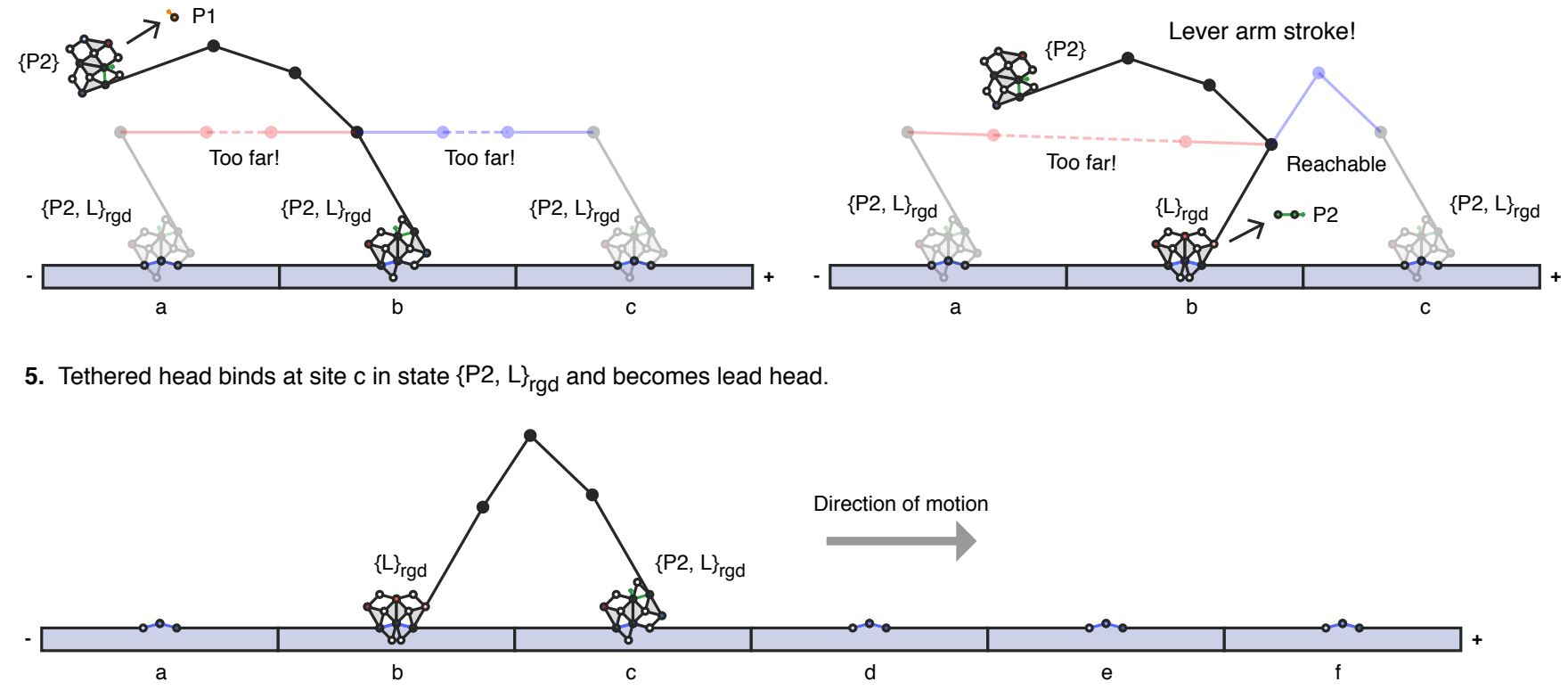

Fig. 26. One step of the ideal-model along a ligand polymer track. 1, The rear head at site $a$, in state $\{\mathrm{L}\}_{\mathrm{rgd}}$, waits for substrate to bind. The front head at site $b$, in state $\{\mathrm{L}, \mathrm{P} 2\}_{\mathrm{rgd}}$, inhibits substrate binding because of P2. 2, Substrate binds to the left side of the rear head placing it in state $\{\mathrm{S}, \mathrm{L}\}$ rgd1. Tension between the heads prevents substrate from binding to the right side, and the head from going through mechanical cycle $2\left(\{\mathrm{~S}, \mathrm{~L}\}_{\mathrm{rgd} 2}\right)$. 3, The rear head dissociates, substrate is cleaved and P1 dissociates, placing the head in state $\{\mathrm{P} 2\}$, now tethered to the bound head. Rebinding of the tethered head at site $a$, in state $\{\mathrm{L}, \mathrm{P} 2\}_{\mathrm{rgd}}$, for the lever arm points in the (-) direction, is now too far for the tether (translucent red; dashed line is the extra length). Binding in front of the bound head, at site $c$, is also too far (translucent blue). 4, The bound head at site $b$ fully binds to the track, displaces P2, and goes through its lever arm stroke. The rotation of the lever arm breaks symmetry and makes site $c$ reachable for the tethered head (translucent blue), and site $a$ more unreachable (translucent red). 5, The tethered head binds at site $c$, reforming the starting configuration, but advanced in the $(+)$ direction along the ligand polymer track.

The landing-bias mechanism is illustrated in Fig. 26 in a sequence of five steps that shows the rear head advancing to the front. The first three steps depict the rear head being displaced from the track and catalysis taking place, leaving the head in state $\{$ P2 $\}$ and tethered to the bound head, which is in state $\{\mathrm{P} 2, \mathrm{~L}\}_{\mathrm{rgd}}($ steps 1-3). Here, both the site the tethered head just left (a), 
and the site in front of the bound head (b), are out of reach (step 3). The landing bias is created when the bound head goes through its lever-arm stroke and breaks mechanical symmetry, placing the tethered head closer to site c (step 4). Chemical symmetry between the bound and tethered head is also broken when P2 is released from the bound head. When the tethered head binds at site $c$, the directional bias created by lever arm stroke of the head at site b, is finally trapped by the dimeric system.

The dimer model illustrates how recognizable motor behavior emerges by connecting more than one TRV allosteric enzyme together - specifically unidirectional motion and head-head coordination. The model also shows how intermolecular tension between the heads is equivalent to directional allosteric control. The connection between interhead tension and allostery is known in all three cytoskeletal motors [12], but the mechanism cannot yet be clearly described. What I show here is that binding at a trivalent binding site can be associated with a transition between two well defined mechanochemical states, which in turn can have a translational effect when the binding sites are oriented parallel to the direction of motion [43]. The translational effect can be used to break directional symmetry if the tether that connects the dimers' heads limits (creates tension) the translational motion required to toggle between the two well defined mechanical states. In this way, a binding state in one head is able to allosterically inhibit the binding state in the other head.

It is important to point out that this mechanism would still work with a flexible, but taught tether connection, rather than the rigid one used in the figures. The main difference is that the translational effect would be smaller. Similar to myosin, directional symmetry is broken at the active sites and is amplified using a rigid extension. In two other models, I use a longer tether between the heads to demonstrate how less ideal coupling can still lead to forward motion (to be included in supplemental material). In both designs the landing bias remains, but the pick-up bias disappears. I show that mechanical cycle 2 can be used to produce forward motion along the track when each enzymatic unit stores the forward bias produced mid-cycle by the other unit, before it reverts.

\section{Conclusion}

The linkage design presented here can in principle be applied to multiple chemistries, including DNA and RNA nanotechnologies [44-47], rotaxane/catenane chemistry [3], and peptide chemistry [48], or a combination of chemistries. While nucleic acid nanotechnologies are currently the most developed for this application [17-20, 49], protein structural design is rapidly advancing [50]. Whichever chemistry is used, the most difficult part would be choosing the catalytic domain, and coupling motion of the catalytic domain to the rest of the structure [51]. RNA enzymes are possibly good candidates [52], because conformational change of RNA can be controlled by tertiary interactions, which is similar to how multivalent binding controls conformational change in the linkage structures here. A protein-based catalytic domain is also possible, but isolating such a domain and being able to mount it within another structure is more challenging than doing the same with nucleic acids. What ATPases do is exactly what is required, but little is known about what makes for a minimal ATPase that can catalyze the breakdown of ATP and nothing else.

Only a small space of allosteric behavior is explored here, which is negative allostery. Even in modeling negative allosteric coupling in myosin, important behavior was left out. The most significant omission from this model is that ligand (actin) binding does not trigger the dissociation 
of the $\mathrm{P}_{\mathrm{i}}$ analog $\mathrm{P} 1$. Including this reaction would require a more complex linkage, and might be a logical next step in extending this model. Intramolecular 'self' binding, between parts of an enzyme, is not explored in this model. Exploring such design with linkages might be interesting, possibly in the context of trying to design cooperative binding or positive allostery. For example, kinesin, which has a neck-linker domain that reversibly binds to its ATPase domain, uses intramolecular 'self' binding of the neck-linker as a form of kinetic control [53].

One shortcoming of the linkage model presented is that the allosteric signals triggered by binding or unbinding are instantaneously felt at the coupled site because the linkages have no slack or floppiness. As the structures grow larger, the speed of information transfer required by the model may be impossibly fast. A solution to this is to allow each bar to be connected to each node by a short flexible tether. This addition would introduce lag time into the flow of mechanical information transfer. And short tether connectors might be one of the few ways to construct flexible junctions with polymers, as seen in some dynamic DNA structures [49].

The geometries I use are two-dimensional and simple compared to the complex three-dimensional geometries seen in proteins and ribonucleoproteins. It is possible that what the linkages represent are allosteric control centers - small dynamic geometries around which larger frameworks can be built that amplify or output the motion of the control center. The idea that conformational changes are amplified or originate from control domains in biomolecular machine is accepted in myosin, the ribosome, and other systems. A major challenge in studying biomolecular machines is figuring out which structures are essential to the function they demonstrate, and which may be nonessential [54-56]. Synthetic systems allow us to start from first principles, using select information we gather from nature. In the case of allostery this is information about the timing of reactions within cycles and the desired behavioral output. In order to use this information to engineer synthetic machines that function allosterically and autonomously, a clear design methodology is needed. The linkage model presented here is intended to be a starting point for creating such a methodology.

[1] Ryota Iino, Kazushi Kinbara, and Zev Bryant. "Introduction: Molecular Motors". en. In: Chem. Rev. 120.1 (Jan. 2020), pp. 1-4.

[2] Heiner Linke et al. "Synthetic biology approaches to dissecting linear motor protein function: towards the design and synthesis of artificial autonomous protein walkers". en. In: Biophys. Rev. 12.4 (Aug. 2020), pp. 1041-1054.

[3] Liang Zhang, Vanesa Marcos, and David A Leigh. "Molecular machines with bio-inspired mechanisms". en. In: Proc. Natl. Acad. Sci. U. S. A. (2018).

[4] Shoshana J Wodak et al. "Allostery in Its Many Disguises: From Theory to Applications". en. In: Structure 27.4 (Apr. 2019), pp. 566-578.

[5] Charles W Carter Jr. "Escapement mechanisms: efficient free energy transduction by reciprocallycoupled gating". en. In: Proteins (Nov. 2019).

[6] J Monod, J Wyman, and J P Changeux. "ON THE NATURE OF ALLOSTERIC TRANSITIONS: A PLAUSIBLE MODEL". In: J. Mol. Biol. 12 (May 1965), pp. 88-118.

[7] Rob Phillips and Nigel Orme. The Molecular Switch: Signaling and Allostery. Princeton University Press, 2020. ISBN: 9780691200248. URL: http://www . jstor . org/stable/ j . ctvx5w8pf.

[8] Aidan I Brown and David A Sivak. "Theory of Nonequilibrium Free Energy Transduction by Molecular Machines". en. In: Chem. Rev. (Aug. 2019). 
[9] R Dean Astumian, Shayantani Mukherjee, and Arieh Warshel. "The Physics and Physical Chemistry of Molecular Machines". en. In: Chemphyschem 17.12 (June 2016), pp. 1719-1741.

[10] Sara Tafoya and Carlos Bustamante. "Molecular switch-like regulation in motor proteins". en. In: Philos. Trans. R. Soc. Lond. B Biol. Sci. 373.1749 (June 2018).

[11] Andreas Ehrmann, Basile Nguyen, and Udo Seifert. "Interlinked GTPase cascades provide a motif for both robust switches and oscillators". en. In: J. R. Soc. Interface 16.157 (Aug. 2019), p. 20190198.

[12] D Thirumalai et al. "Symmetry, Rigidity, and Allosteric Signaling: From Monomeric Proteins to Molecular Machines". en. In: Chem. Rev. (2019).

[13] Holger Flechsig. "Design of Elastic Networks with Evolutionary Optimized Long-Range Communication as Mechanical Models of Allosteric Proteins". en. In: Biophys. J. 113.3 (Aug. 2017), pp. 558-571.

[14] Jason W Rocks et al. "Designing allostery-inspired response in mechanical networks". en. In: Proc. Natl. Acad. Sci. U. S. A. 114.10 (Mar. 2017), pp. 2520-2525.

[15] Le Yan et al. "Architecture and coevolution of allosteric materials". en. In: Proc. Natl. Acad. Sci. U. S. A. 114.10 (Mar. 2017), pp. 2526-2531.

[16] Erik D. Demaine and Joseph O'Rourke. Geometric Folding Algorithms: Linkages, Origami, Polyhedra. Reprint. USA: Cambridge University Press, 2008. IsBN: 9780521715225.

[17] W Shen et al. "A protein-driven DNA device that measures the excess binding energy of proteins that distort DNA". In: ANGEWANDTE CHEMIE-INTERNATIONAL EDITION IN ENGLISH- 43 (Jan. 2004), pp. 4750-4752.

[18] Chao Zhou, Zhongqiang Yang, and Dongsheng Liu. "Reversible regulation of protein binding affinity by a DNA machine". en. In: J. Am. Chem. Soc. 134.3 (Jan. 2012), pp. 1416-1418.

[19] Minghui Liu et al. "A DNA tweezer-actuated enzyme nanoreactor". In: Nat. Commun. 4 (2013), p. 2127.

[20] Yonggang Ke et al. "Regulation at a distance of biomolecular interactions using a DNA origami nanoactuator". en. In: Nat. Commun. 7 (Mar. 2016), p. 10935.

[21] William P Jencks. "From Chemistry to Biochemistry to Catalysis to Movement". In: Annu. Rev. Biochem. 66.1 (1997), pp. 1-18.

[22] E Branscomb et al. "Escapement mechanisms and the conversion of disequilibria; the engines of creation". In: Phys. Rep. 677.Supplement C (2017), pp. 1-60.

[23] Matthias Preller and Dietmar J Manstein. "Myosin structure, allostery, and mechano-chemistry". en. In: Structure 21.11 (Nov. 2013), pp. 1911-1922.

[24] Julien Robert-Paganin et al. "Force Generation by Myosin Motors: A Structural Perspective". en. In: Chem. Rev. (Nov. 2019).

[25] Pinar S Gurel et al. "Cryo-EM structures reveal specialization at the myosin VI-actin interface and a mechanism of force sensitivity". en. In: Elife 6 (Dec. 2017).

[26] Julian von der Ecken et al. "Cryo-EM structure of a human cytoplasmic actomyosin complex at near-atomic resolution". en. In: Nature 534.7609 (June 2016), pp. 724-728.

[27] F A Kiani and S Fischer. "Catalytic strategy used by the myosin motor to hydrolyze ATP". In: Proc. Natl. Acad. Sci. U. S. A. 111.29 (July 2014), E2947-E2956.

[28] Farooq Ahmad Kiani and Stefan Fischer. "ATP-dependent interplay between local and global conformational changes in the myosin motor". en. In: Cytoskeleton 73.11 (Nov. 2016), pp. 643651. 
[29] Ahmet Mentes et al. "High-resolution cryo-EM structures of actin-bound myosin states reveal the mechanism of myosin force sensing". en. In: Proc. Natl. Acad. Sci. U. S. A. 115.6 (Feb. 2018), pp. 1292-1297.

[30] Bernard Yurke et al. "A DNA-fuelled molecular machine made of DNA". In: Nature 406.6796 (Aug. 2000), pp. 605-608.

[31] T L Hill and E Eisenberg. "Can free energy transduction be localized at some crucial part of the enzymatic cycle?" en. In: Q. Rev. Biophys. 14.4 (Nov. 1981), pp. 463-511.

[32] Carlo Fasting et al. "Multivalency as a chemical organization and action principle". en. In: Angew. Chem. Int. Ed Engl. 51.42 (2012), pp. 10472-10498.

[33] Tine Curk, Jure Dobnikar, and Daan Frenkel. "Design Principles for Super Selectivity using Multivalent Interactions". In: Multivalency. Ed. by Jurriaan Huskens et al. Vol. 40. Chichester, UK: John Wiley \& Sons, Ltd, Jan. 2018, pp. 75-101.

[34] Niranjan Srinivas et al. "On the biophysics and kinetics of toehold-mediated DNA strand displacement". In: Nucleic Acids Res. 41.22 (Dec. 2013), pp. 10641-10658.

[35] Timo R Maarleveld, Brett G Olivier, and Frank J Bruggeman. "StochPy: a comprehensive, user-friendly tool for simulating stochastic biological processes". en. In: PLoS One 8.11 (2013), e79345.

[36] P D Boyer. "A perspective of the binding change mechanism for ATP synthesis". In: FASEB J. 3.10 (Aug. 1989), pp. 2164-2178.

[37] M Caplow, R L Ruhlen, and J Shanks. "The Free Energy for Hydrolysis of a MicrotubuleBound Nucleotide Tripllosphate Is Near Zero: All of the Free Energy for Hydrolysis Is Stored in the Microtubule Lattice". In: J. Cell Biol. 127.3 (1994).

[38] E M De La Cruz, A L Wells, and S S Rosenfeld. "The kinetic mechanism of myosin V". In: ... of Sciences of the ... (1999).

[39] Andrew J Turberfield et al. "DNA fuel for free-running nanomachines". In: Phys. Rev. Lett. 90.11 (Mar. 2003), p. 118102.

[40] Sebastian Kühner and Stefan Fischer. "Structural mechanism of the ATP-induced dissociation of rigor myosin from actin". en. In: Proc. Natl. Acad. Sci. U. S. A. 108.19 (May 2011), pp. 7793-7798.

[41] Mary Williard Elting et al. "Detailed Tuning of Structure and Intramolecular Communication Are Dispensable for Processive Motion of Myosin VI". In: Biophys. J. 100.2 (Jan. 2011), pp. $430-439$.

[42] R Dean Astumian. "Thermodynamics and kinetics of molecular motors". In: Biophys. J. 98.11 (June 2010), pp. 2401-2409.

[43] Zhisong Wang. "Synergic mechanism and fabrication target for bipedal nanomotors". In: Proc. Natl. Acad. Sci. U. S. A. 104.46 (Nov. 2007), pp. 17921-17926.

[44] Nadrian C Seeman and Hanadi F Sleiman. "DNA nanotechnology". In: Nature Reviews Materials 3.1 (Nov. 2017), p. 17068.

[45] Swarup Dey et al. "DNA origami". In: Nature Reviews Methods Primers 1.1 (Jan. 2021), p. 13.

[46] Joseph D Yesselman et al. "Computational design of three-dimensional RNA structure and function". en. In: Nat. Nanotechnol. 14.9 (Sept. 2019), pp. 866-873.

[47] Wade W Grabow and Luc Jaeger. "RNA self-assembly and RNA nanotechnology". en. In: Acc. Chem. Res. 47.6 (June 2014), pp. 1871-1880. 
[48] Po-Ssu Huang, Scott E Boyken, and David Baker. "The coming of age of de novo protein design". en. In: Nature 537.7620 (Sept. 2016), pp. 320-327.

[49] Jie Song et al. "Reconfiguration of DNA molecular arrays driven by information relay". en. In: Science 357.6349 (July 2017).

[50] Ariel J Ben-Sasson et al. "Design of biologically active binary protein 2D materials". en. In: Nature 589.7842 (Jan. 2021), pp. 468-473.

[51] Hamid Ramezani and Hendrik Dietz. "Building machines with DNA molecules". In: Nat. Rev. Genet. (Oct. 2019).

[52] Cedric Reymond, Jean-Denis Beaudoin, and Jean-Pierre Perreault. "Modulating RNA structure and catalysis: lessons from small cleaving ribozymes". In: Cell. Mol. Life Sci. 66.24 (Dec. 2009), pp. 3937-3950.

[53] R A Cross. "Review: Mechanochemistry of the kinesin-1 ATPase". In: Biopolymers 105.8 (Aug. 2016), pp. 476-482.

[54] Michael Manhart and Alexandre V Morozov. "Protein folding and binding can emerge as evolutionary spandrels through structural coupling". en. In: Proc. Natl. Acad. Sci. U. S. A. 112.6 (Feb. 2015), pp. 1797-1802.

[55] Olivier Rivoire. "Parsimonious evolutionary scenario for the origin of allostery and coevolution patterns in proteins". en. In: Phys Rev E 100.3-1 (Sept. 2019), p. 032411.

[56] Georg K A Hochberg et al. "A hydrophobic ratchet entrenches molecular complexes". en. In: Nature (Dec. 2020).

\section{Acknowledgements}

I thank David Soloveichik, Zev Bryant, Keenan Breik, Erik Winfree, Muneaki Nakamura, Dean Astumian, and Enrique Rojas for helpful discussions about the draft and ideas within. I thank David Soloveichik for crucial discussions early on that helped push the concepts forward and helped with running simulations. I thank Zev Bryant for crucial discussions about molecular motors and the walker models proposed in the paper, and for encouraging me to include the DIV model. This work was supported by a National Institutes of Health (NIH) Fellowship F32GM09442 to Tosan Omabegho, while at Stanford University. 